\title{
Comparison of the response to geometrical complexity of methods for unstationary simulations in discrete fracture networks with conforming, polygonal, and non-matching grids
}

\author{
Andrea Borio $^{1}$. Alessio Fumagalli ${ }^{1,2}$ (D) . Stefano Scialò ${ }^{1}$
}

Received: 11 August 2019 / Accepted: 6 August 2020 / Published online: 23 October 2020

(C) The Author(s) 2020

\begin{abstract}
The aim of this study is to compare numerical methods for the simulation of single-phase flow and transport in fractured media, described here by means of the discrete fracture network (DFN) model. A Darcy problem is solved to compute the advective field, then used in a subsequent time-dependent transport-diffusion-reaction problem. The numerical schemes are benchmarked in terms of flexibility in handling geometrical complexity, mass conservation, and stability issues for advection-dominated flow regimes. To this end, two benchmark cases, along with an additional one from a previous work, have been specifically designed and are here proposed and investigated, representing some of the most critical issues encountered in DFN simulations.
\end{abstract}

Keywords Discrete fracture network · Benchmark · Discretization methods · Domain decomposition · Non-matching grids · Polygonal grids

Mathematics Subject Classification (2010) 02.60.Cb $\cdot 02.60 . \mathrm{Lj} \cdot 02.70 . \mathrm{Dh}$

\section{Introduction}

The movement of liquids in the underground is heavily influenced by the presence of fractures and their relative intersections [25, 27, 28, 30, 41]. Fractures are discontinuities (here assumed planar) along which a rock has been broken, mainly due to geological movements or to artificial stimulation [48]. In this work, we are considering only open structures, characterized by a geometrical aperture,

Members of the INdAM research group GNCS.

Andrea Borio

andrea.borio@polito.it

Alessio Fumagalli

alessio.fumagalli@polito.it; alessio.fumagalli@polimi.it

Stefano Scialò

stefano.scialo@polito.it

1 Dipartimento di Scienze Matematiche, Politecnico di Torino, Corso Duca degli Abruzzi 24, 10129 Torino, Italy

2 Present address: MOX - Dipartimento di Matematica

"F. Brioschi", Politecnico di Milano, via Bonardi 9, 20133 Milan, Italy that allow a liquid to flow through [28]. Possibilities are actual fractures, faults, and joints. We are thus excluding low permeable (closed/impervious) objects such as veins or dykes. For particular underground compositions (e.g., granite, shale, or sandstone), the rock permeability is several orders of magnitude smaller than the fracture permeability. It is a common choice and a reasonable approximation to ignore the rock matrix effect in the simulations and rely only on the fractures. The framework is the discrete fracture network model (DFN), where the aperture is not a geometrical constraint but a parameter in the bidimensional representation of the fractures by reduced models; see [47] and the forthcoming references.

The geometrical complexity of natural fracture networks may impose difficulties in the numerical simulations, due to the presence of small intersections between fractures, different intersection configurations (e.g., Y-type or L-type), small angles of intersection, and small distances between intersection lines. In the literature, three main approaches are developed to overcome these difficulties.

The first approach considers rather standard numerical scheme for the discretization of the physical equations, and relies on robust grid generation software. The coupling conditions among fractures are imposed via Lagrange multipliers on a representation of the interfaces conforming 
with the computational mesh on all sides. To be specific, the edges of the computational elements have to match at the intersections. This approach, called conforming-mesh discretization, may suffer, for example, when intersections lines are very close to each other; see [26, 42, 43, 49, 50]. To partially overcome these problems, a possibility is to consider the class of polytopal methods, like mimetic finite difference and polytopic discontinuous Galerkin (DG) that allow grid elements of general shapes. Numerical methods of this type are introduced in $[2,3,7,9,10,29,34,35]$. A recent work [58] extends the use of the enriched Galerkin (EG) approach [46] to mixed-dimensional problems in porofractured domains.

A second possibility is to keep the intersections explicitly represented, but relaxing the conformity of the edges. This approach, named non-conforming mesh discretization, requires more advanced numerical schemes based on the mortar technique. In this case, we relax the actual generation of the fracture meshes which usually gives fewer discrete elements than the conforming mesh approach $[6,22,23$, 51-53]. This method may still suffer in the presence of severe geometries. Also, in this case, the virtual element methods are an interesting option to further decrease the computational cost; see [60, 62].

A third family of schemes comprises the so-called nonmatching-mesh discretizations. In terms of mesh generation, in this case, the intersections do not place any constraints, as the fractures are meshed independently and the coupling conditions are imposed by an optimization procedure. A functional that measures the mismatch in the coupling conditions is minimized iteratively, where only the degrees of freedom involved in the intersections (the cut region) are considered [5, 16-19]. This procedure is independent on the actual numerical schemes, which might take advantage of ad hoc strategies to enrich the solution in the cut region. Extended finite elements are a successful example; see, for example, [24, 31, 38, 39]. An alternative, based on the cut finite element method, is proposed in [21].

The present work extends and enriches the one proposed in [37] to more complex physical phenomena. The concepts previously discussed are applied to Darcy and heat transport-and-diffusion models. The Darcy velocity is computed first and then used as an advective field for the heat equation. High-quality computation or reconstruction of the Darcy velocity may significantly impact the temperature distribution. Moreover, in the heat equation, the transport part usually dominates the diffusion (Péclet number greater than 1) and stable or stabilized schemes are needed to avoid or limit spurious oscillations that might compromise the accuracy of the solution. In the numerical tests, we are considering several numerical schemes, a subset of the previously cited papers that are in the expertise of this work authors, for the comparison to cover most of the combinations discussed and try to assess their performance. The aim of this work is thus twofold: establish a set of benchmark cases and give guidance in the development of more advanced numerical schemes to solve this problem.

The paper is organized as follows. In Section 2, the Darcy and heat models are introduced and discussed, with particular focus on the coupling conditions. Section 3 is devoted to the description of the proposed numerical schemes. Three numerical examples are presented and discussed in Section 4, comparing the performances of the considered numerical schemes. Finally, in Section 5, we draw some conclusions and suggestions for future developments.

\section{Mathematical model}

In this section, we introduce the mathematical model used to describe the hydraulic head and Darcy velocity profiles in a discrete fracture network. Once this problem is solved, the Darcy velocity is considered an advective field to simulate the transport and diffusion of heat in DFNs.

Fractures are considered non-overlapping planar polygons, which can be connected to other fractures through intersection segments, also called traces. We consider $N_{\Omega}$ fractures $\Omega_{i} \subset \mathbb{R}^{3}$ with boundary $\partial \Omega_{i}$, which compose the discrete fracture network $\Omega=\cup_{i=1}^{N_{\Omega}} \Omega_{i}$, and we denote its boundary as $\partial \Omega$ with outward unit normal $\boldsymbol{n}_{\text {ext }}$, defined on each fracture plane as the unit vector normal to the fracture boundary pointing outward from the fracture polygon.

Given two distinct and intersecting fractures $\Omega_{i}$ and $\Omega_{j}$, with $i \neq j$, we denote their intersection (trace) by $\overline{\Gamma_{k}}=$ $\overline{\Omega_{i}} \cap \overline{\Omega_{j}}$. For simplicity, we assume that a trace is formed only by two distinct fractures; however, this assumption can be relaxed. A natural order of indexes can be introduced to numerate the traces $\Gamma_{k}$ from 1 to $N_{\Gamma}$, the latter being their cardinality. We consider also the function $t:\left\{1, \ldots, N_{\Omega}\right\} \times$ $\left\{1, \ldots, N_{\Omega}\right\} \rightarrow\left\{1, \ldots, N_{\Gamma}\right\}$ such that $k=t(i, j)$ with $\overline{\Gamma_{k}}=\overline{\Omega_{i}} \cap \overline{\Omega_{j}}$. We have $t(i, j)=t(j, i)$ and its inverse $t^{-1}$ is well defined such that $(i, j)=t^{-1}(k)$ where $i<j$. We denote by $\Gamma=\cup_{k=1}^{N_{\Gamma}} \Gamma_{k}$ the union of all the traces and by $\Gamma_{\Omega_{i}}$ the set of traces belonging to the fracture $\Omega_{i}$. Moreover, consider a fracture $\Omega_{i}$ and a trace $\Gamma_{k}$, with $k \in \Gamma_{\Omega_{i}}$. $\Gamma_{k}$ naturally subdivides $\Omega_{i}$ in two sub-regions, indicated by $\Omega_{i,+}^{k}$ and $\Omega_{i,-}^{k}$, such that $\Gamma_{k} \subset\left(\partial \Omega_{i,+}^{k} \cap \partial \Omega_{i,-}^{k}\right)$. To each of these sub-regions, we associate an outward unit normal perpendicular to $\Gamma_{k}$, denoted by $\boldsymbol{n}_{i,+}$ and $\boldsymbol{n}_{i,-}$ (with $\boldsymbol{n}_{i,+}=$ $\left.-\boldsymbol{n}_{i,-}\right)$ and a trace operator, respectively denoted by $\operatorname{tr}_{i,+}^{k}$ and $\operatorname{tr}_{i,-}^{k}$. Generic trace operators on fracture $\Omega_{i}$ are denoted by $\operatorname{tr}_{i}$. An example of a simple DFN with the introduced nomenclature is given in Fig. 1.

We present the Darcy problem in strong and weak form in Section 2.1, to compute the hydraulic head and Darcy 


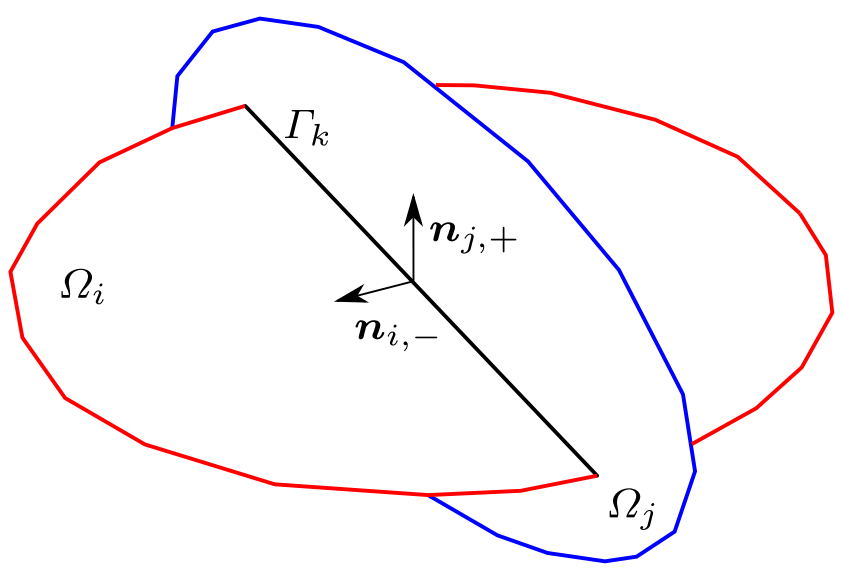

Fig. 1 Representation of two fractures $\Omega_{i}$ and $\Omega_{j}$ intersecting in $\Gamma_{k}$

velocity, whereas the heat equation is introduced along with its weak formulation and functional setting in Section 2.2.

\subsection{The Darcy model}

This section is devoted to a brief presentation of the mathematical models used to describe the hydraulic head field $h$ and Darcy velocity $\boldsymbol{u}$ : further details being available in $[16,17,34,37]$ and references therein. Unknowns and parameters restricted to a fracture $\Omega_{i}$ are denoted by a subscript $i$.

For clarity in the exposition, we start considering a single fracture $\Omega_{i}$. The Darcy model on $\Omega_{i}$ reads: find $\left(\boldsymbol{u}_{i}, h_{i}\right)$ such that:

$$
\begin{aligned}
& \boldsymbol{u}_{i}+K_{i} \nabla h_{i}=\mathbf{0} \\
& \nabla \cdot \boldsymbol{u}_{i}=f_{i}
\end{aligned} \quad \text { in } \Omega_{i} \backslash \Gamma
$$

Variables, data, and differential operators are defined on the tangent plane of the fracture. $K_{i}$ is the effective hydraulic conductivity tensor, which is symmetric and positive definite, and $f_{i}$ is a scalar source/sink term. In our applications, following lubrication theory [4, 56, 63], we consider the intrinsic fracture permeability, obtained by the cubic law, scaled by the aperture: $k_{i}=\epsilon_{i}^{2} / 12$, where $\epsilon_{i}$ is the fracture aperture. Moreover, the effective hydraulic conductivity in Eq. (1a) is isotropic and defined as:

$K_{i}=\frac{\epsilon_{i} k_{i} \rho_{w} g}{\mu} I$,

with $\rho_{w}$ the fluid density, $g$ the gravity acceleration, and $\mu$ the dynamic viscosity of the fluid.

The boundary conditions on $\Omega_{i}$ are:

$$
\begin{array}{ll}
\operatorname{tr}_{i}^{\partial} h_{i}=\bar{h}_{i} & \text { on } \partial \Omega_{i, D} \\
\operatorname{tr}_{i}^{\partial} \boldsymbol{u}_{i} \cdot \boldsymbol{n}_{\text {ext }}=0 & \text { on } \partial \Omega_{i, N}
\end{array}
$$

where $\partial \Omega_{i, D}$ and $\partial \Omega_{i, N}$ are a partition of $\partial \Omega_{i}$ and $\operatorname{tr}_{i}^{\partial}$ is the trace operator on $\partial \Omega_{i}$. The boundary conditions considered here are chosen to be coherent with the examples in Section 4 . The generalization to other boundary conditions is straightforward. We assume that $\partial \Omega_{i, D} \neq \varnothing$ for at least one $i$. Equations (1a) and (1b) are well studied in the literature; refer to the aforementioned references.

Let us now consider two distinct fractures $\Omega_{i}$ and $\Omega_{j}$ forming an intersection $\Gamma_{k}$. On both fractures (1a) and (1b) are considered and we assume continuous coupling conditions for the hydraulic head and the normal component of the Darcy velocity at the trace. The coupling conditions between two fractures $\Omega_{i}$ and $\Omega_{j}$ such that $t(i, j)=k$, read:

$$
\begin{array}{ll}
\sum_{l \in\{i, j\}} \operatorname{tr}_{l,+}^{k} \boldsymbol{u}_{l} \cdot \boldsymbol{n}_{l,+}+\operatorname{tr}_{l,-}^{k} \boldsymbol{u}_{l} \cdot \boldsymbol{n}_{l,-}=0 & \\
\operatorname{tr}_{i,+}^{k} h_{i}=\operatorname{tr}_{i,-}^{k} h_{i}=\operatorname{tr}_{j,+}^{k} h_{j}=\operatorname{tr}_{j,-}^{k} h_{j} & \text { on } \Gamma_{k} .
\end{array}
$$

The case of multiple fractures follows immediately from our assumptions. The Darcy equation can be formalized in the following problem.

Problem 1 (Darcy equation on a DFN-mixed formulation) Given the set of fractures $\Omega$ and traces $\Gamma$, find $(\boldsymbol{u}, h)$ such that (1a)-(1b)-(1c) are satisfied.

Let $Q=\bigoplus_{i=1}^{N_{\Omega}} L^{2}\left(\Omega_{i}\right)$ and suppose $f_{i} \in L^{2}\left(\Omega_{i}\right)$, $K_{i} \in L^{\infty}\left(\Omega_{i}\right)$ and $\bar{h}_{i} \in H^{\frac{1}{2}}\left(\Omega_{i}\right), \forall i$. It is known that solving Problem 1 is equivalent to solve one of the following two weak problems, corresponding to the mixed and primal formulations, respectively.

Problem 2 (Darcy equation on a DFN-mixed weak formulation) Let us define the functional space $V$ as

$$
\begin{array}{r}
V=\left\{\boldsymbol{v}: \boldsymbol{v}_{i} \in H_{\nabla \cdot(}\left(\Omega_{i}\right), \operatorname{tr}_{i}^{\partial} \boldsymbol{v}_{i} \cdot \boldsymbol{n}_{\mathrm{ext}}=0 \text { on } \partial \Omega_{i, N} \forall i,\right. \\
\sum_{l \in\{i, j\}} \operatorname{tr}_{l,+}^{k} \boldsymbol{u}_{l} \cdot \boldsymbol{n}_{l,+}+\operatorname{tr}_{l,-}^{k} \boldsymbol{u}_{l} \cdot \boldsymbol{n}_{l,-}=0 \\
\left.\forall k, t^{-1}(k)=(i, j)\right\} .
\end{array}
$$

Then, the problem reads: find $(\boldsymbol{u}, h) \in V \times Q$ such that

$$
\left\{\begin{array}{cc}
\sum_{i=1}^{N_{\Omega}}\left(K_{i}^{-\frac{1}{2}} \boldsymbol{u}, K_{i}^{-\frac{1}{2}} \boldsymbol{v}\right)_{\Omega_{i}}-\sum_{i=1}^{N_{\Omega}}(\nabla \cdot \boldsymbol{v}, h)_{\Omega_{i}} & \\
=-\sum_{i=1}^{N_{\Omega}}\left\langle\bar{h}_{i}, \operatorname{tr}_{i}^{\partial} \boldsymbol{v} \cdot \boldsymbol{n}\right\rangle_{\partial \Omega_{i, D}} & \\
-\sum_{i=1}^{N_{\Omega}}(\nabla \cdot \boldsymbol{u}, q)_{\Omega_{i}}=\sum_{i=1}^{N_{\Omega}}\left(f_{i}, q\right)_{\Omega_{i}} & \forall q \in Q .
\end{array}\right.
$$

Problem 3 (Darcy equation on a DFN-primal weak formulation) Let us define the functional space $V$ as

$$
\begin{array}{r}
V=\left\{v: v \in H^{1}\left(\Omega_{i}\right), \operatorname{tr}_{i}^{\partial} v=0 \text { on } \partial \Omega_{i, D} \forall i\right. \\
\operatorname{tr}_{i,+}^{k} v_{i}=\operatorname{tr}_{i,-}^{k} v_{i}=\operatorname{tr}_{j,+}^{k} v_{j}=\operatorname{tr}_{j,-}^{k} v_{j}, \\
\left.\forall k, t^{-1}(k)=(i, j)\right\} .
\end{array}
$$


Then the problem reads: find $h \in V$ such that

$$
\begin{aligned}
\sum_{i=1}^{N_{\Omega}}\left(K_{i}^{\frac{1}{2}} \nabla h, K_{i}^{\frac{1}{2}} \nabla v\right)_{\Omega_{i}} & =\sum_{i=1}^{N_{\Omega}}\left(f_{i}, v\right)_{\Omega_{i}} \\
& -\sum_{i=1}^{N_{\Omega}}\left(K_{i}^{\frac{1}{2}} \nabla h_{D, i}, K_{i}^{\frac{1}{2}} \nabla v\right)_{\Omega_{i}} \quad \forall v \in V,
\end{aligned}
$$

where $h_{D, i} \in H^{1}\left(\Omega_{i}\right) \forall i$ is a lifting of $\bar{h}_{i}$.

\subsection{The heat equation}

In this part, we present the heat equation on the DFN. Once problem 1 is solved, the Darcy velocity can be used as advective field in the transport problem. We denote the temperature in a DFN as $\theta$, and its restriction to fracture $\Omega_{i}$ as $\theta_{i}$. The heat equation on $\Omega_{i}$ reads: given $\boldsymbol{u}_{i}$ find $\theta_{i}$ such that

$$
\begin{cases}\zeta_{i} \partial_{t} \theta_{i}+\nabla \cdot\left(\boldsymbol{u}_{i} \theta_{i}-D_{i} \nabla \theta_{i}\right) & \text { in } \Omega_{i} \backslash \Gamma \times(0, T], \\ +\iota_{i}\left(\theta_{i}-\hat{\theta}_{i}\right)=0 & \text { in } \Omega_{i} \backslash \Gamma,\end{cases}
$$

where $T \in \mathbb{R}$ is the end time of the simulation. Also, in this case, the variables, data, and differential operators are defined on the tangent plane of the fracture. The relations to compute the physical parameters are $[4,56,63]$ :

$\zeta_{i}=\frac{\epsilon_{i} c_{e, i}}{\rho_{w} c_{w}}, \quad D_{i}=\frac{\epsilon_{i} \lambda_{e, i}}{\rho_{w} c_{w}}, \quad \iota_{i}=\frac{\gamma_{e, i}}{\rho_{w} c_{w}}$,

where $\epsilon_{i}$ is the fracture aperture, $\rho_{w}$ the fluid density, $c_{w}$ the fluid specific thermal capacity, $c_{e, i}=\phi_{i} \rho_{w} c_{w}+$ $\left(1-\phi_{i}\right) \rho_{m} c_{m}$ the fracture effective thermal capacity, $\phi_{i}$ the fracture porosity, $\rho_{m}$ the density of the rock matrix, $c_{m}$ the specific thermal capacity of the rock matrix, $\lambda_{e, i}=\lambda_{w}^{\phi_{i}} \lambda_{m}^{1-\phi_{i}}$ the effective thermal conductivity, $\gamma_{e, i}$ the effective heat transfer coefficient between fluid and rock, and, finally, $\hat{\theta}_{i}$ is the temperature of the rock matrix, acting as external heat source/sink.

For brevity, we present only boundary conditions conforming to the numerical tests of Section 4. Recalling that $\partial \Omega_{i, D}$ is the Dirichlet portion of the boundary of fracture $\Omega_{i}$ for the Darcy problem in Section 2.1, let us split $\partial \Omega_{i, D}$ into two parts, namely $\partial \Omega_{i, D}^{\text {inflow }}$ and $\partial \Omega_{i, D}^{\text {outflow }}$. Thus, according to the computed Darcy velocity $\boldsymbol{u}_{i}$, on each fracture $\Omega_{i}$, the inflow boundary $\partial \Omega_{i, D}^{\text {inflow }}$ is the portion of $\partial \Omega_{i, D}$ where $\boldsymbol{u}_{i} \cdot \boldsymbol{n}_{\text {ext }}<0$ and conversely the outflow boundary is the portion of $\partial \Omega_{i, D}$ where $\boldsymbol{u}_{i} \cdot \boldsymbol{n}_{\text {ext }}>0$, thus linking the nature of the boundary to the solution of the Darcy problem. Note that $\partial \Omega_{i, D}^{\text {inflow }}$ and $\partial \Omega_{i, D}^{\text {outflow }}$ might be both empty for most of the fractures. Then, boundary conditions on fracture $\Omega_{i}$ are:

$$
\begin{array}{ll}
\operatorname{tr}_{i}^{\partial} \theta_{i}=\bar{\theta}_{i} & \text { on } \partial \Omega_{i, D}^{\text {inflow }} \times(0, T], \\
\operatorname{tr}_{i}^{\partial} D_{i} \nabla \theta_{i} \cdot \boldsymbol{n}_{\text {ext }}=0 & \text { on } \partial \Omega_{i, D}^{\text {outflow }} \times(0, T], \\
\operatorname{tr}_{i}^{\partial} D_{i} \nabla \theta_{i} \cdot \boldsymbol{n}_{\text {ext }}=0 & \text { on } \partial \Omega_{i, N} \times(0, T] .
\end{array}
$$

Note that, by Eq. (1b), $\operatorname{tr}_{i}^{\partial}\left(D_{i} \nabla \theta_{i}+\boldsymbol{u}_{i} \theta\right) \cdot \boldsymbol{n}_{\text {ext }}=0$ on $\partial \Omega_{i, N}$.

The coupling conditions between two fractures $\Omega_{i}$ and $\Omega_{j}$ such that $t(i, j)=k$, read, $\forall t \in(0, T]$,

$$
\begin{aligned}
& \sum_{l \in\{i, j\}} \operatorname{tr}_{l,+}\left(\boldsymbol{u}_{l} \theta_{l}-D_{l} \nabla \theta_{l}\right) \cdot \boldsymbol{n}_{l,+} \\
& \quad+\operatorname{tr}_{l,-}\left(\boldsymbol{u}_{l} \theta_{l}-D_{l} \nabla \theta_{l}\right) \cdot \boldsymbol{n}_{l,-}=0, \\
& \operatorname{tr}_{i,+} \theta_{i}=\operatorname{tr}_{i,-} \theta_{i}=\operatorname{tr}_{j,+} \theta_{j}=\operatorname{tr}_{j,-} \theta_{j} .
\end{aligned}
$$

The heat equation can be formalized in the following problem.

Problem 4 (Heat equation on a DFN) Given the set of fractures $\Omega$ and traces $\Gamma$ and the Darcy velocity $\boldsymbol{u}$, find $\theta$ such that $(2 \mathrm{a})-(2 \mathrm{~b})-(2 \mathrm{c})$ are satisfied.

We consider the following primal weak formulation of problem 4.

Problem 5 (Heat equation on a DFN-weak formulation) Suppose $\zeta_{i}, D_{i}, \iota_{i} \in L^{\infty}\left(\Omega_{i}\right)$ and $\hat{\theta}_{i} \in L^{2}\left(\Omega_{i}\right), \forall i$ and let us define, for any $\Upsilon$ such that $\Upsilon_{i} \in H^{\frac{1}{2}}\left(\partial \Omega_{i, D}^{\text {inflow }}\right)$,

$$
\begin{gathered}
W_{\Upsilon}=\left\{v: v_{i} \in L^{2}\left(0, T ; H^{1}\left(\Omega_{i}\right)\right),\right. \\
\operatorname{tr}_{i}^{\partial} v(t)=\Upsilon_{i} \text { on } \partial \Omega_{i, D}^{\text {inflow }} \forall i, \forall t \in(0, T), \\
\operatorname{tr}_{i,+}^{k} v_{i}(t)=\operatorname{tr}_{i,-}^{k} v_{i}(t)=\operatorname{tr}_{j,+}^{k} v_{j}(t)=\operatorname{tr}_{j,-}^{k} v_{j}(t), \\
\left.\forall t \in(0, T], \forall k, t^{-1}(k)=(i, j)\right\}
\end{gathered}
$$

The problem reads: find $\theta \in W_{\bar{\theta}}$ such that $\theta_{i}(0)=\overline{\overline{\theta_{i}}} \forall i$ and, $\forall v \in W_{0}$,

$$
\begin{aligned}
& \int_{0}^{T} \sum_{i=1}^{N_{\Omega}}\left[-\left(\zeta_{i} \partial_{t} \theta, v\right)_{\Omega_{i}}+\left(D_{i}^{\frac{1}{2}} \nabla \theta, D_{i}^{\frac{1}{2}} \nabla v\right)_{\Omega_{i}}\right. \\
& \left.+\left(\nabla \cdot\left(\boldsymbol{u}_{i} \theta\right), v\right)_{\Omega_{i}}+\left(\iota_{i} \theta, v\right)_{\Omega_{i}}\right]=\int_{0}^{T} \sum_{i=1}^{N_{\Omega}}\left(\iota_{i} \hat{\theta}_{i}, v\right)_{\Omega_{i}}
\end{aligned}
$$

\section{Numerical discretization}

In this section, we present various discretization strategies, both well-established, both unconventional, that can be used to solve the models described in Section 2. These strategies have similarities and differences that can be used to categorize them. A first point concerns the computational mesh and, in particular, how the meshing is performed at fracture intersections: it is possible to have a matching or 
non-matching grids. In the former case, fracture grids are conforming to the intersections among fractures, while in the latter, grid elements arbitrarily cross intersections. A second issue is related to mass conservation: in computing the Darcy velocity, some schemes are locally mass conservative and some other are only globally conservative, and this property may impact the subsequent solution of the heat problem. Also, some numerical schemes are characterized by high numerical diffusivity which might impact the solution but also avoids unphysical spurious oscillations in advection dominated flow regimes, whereas other schemes need to adopt stabilization techniques.

Six different approaches are considered in the present work, given as the combination of a numerical scheme for the computation of the Darcy velocity and a numerical scheme for the spatial semi-discretization of the subsequent non-stationary advection-diffusion-reaction problem (shortly denoted as heat equation). The implicit Euler method is used, instead, in all cases, for time evolution. The impact of different time advancing schemes is also of interest but is not considered here, the focus being the geometrical complexity and thus the spatial discretization techniques. The approaches are listed in Table 1. The scheme tagged TPFAUP is given by the combination of the two-point flux approximation (TPFA) method for the Darcy problem and the TPFA with upwinding for the advection term (TPFA+UPWIND) for the heat equation. Scheme MFEMUP uses instead mixed finite elements (MFEM) for the Darcy equation and again TPFA + UPWIND for the heat equation. The method MVEMUP uses instead the virtual element method in mixed formulation (MVEM) for the Darcy problem, on matching polygonal meshes. These schemes are implemented in PorePy, a simulation tool written in Python for fractured and deformable porous media; see $[44,45]$. PorePy is freely available on GitHub along with the numerical tests proposed in Section 4. The method labeled MFEMSUPG is based on mixed standard finite elements for the numerical resolution of the Darcy problem and on standard finite elements (FEM) with streamline upwind Petrov-Galerkin (SUPG) stabilization [32] for the heat equation. Finally, methods FEMSUPG and XFEMSUPG use a non-conventional optimization-based approach for the Darcy equation and with SUPG stabilization also for the heat equation. The optimization approach can adopt different baseline discretization methods: here, we consider the variants using standard finite elements (OPT-FEM) and extended finite elements (OPT-XFEM). Methods MFEMSUPG, FEMSUPG and XFEMSUPG are implemented in C++ and Matlab ${ }^{\circledR}$.

The forthcoming parts describe in more detail the previous approaches, grouping them according to the coupling at the traces: matching coupling at traces in Section 3.1 and non-matching coupling in Section 3.2.

\subsection{Matching discretization at traces}

Here, advantages and drawbacks of a conforming mesh discretization at the traces are discussed. As mentioned before in a conforming grid, the meshes of both the intersecting fractures match the trace with their geometry. The trace is thus entirely covered by contiguous cell edges of the two fractures; see Fig. 2a and b as an example. This approach has the clear advantage of an easy applicability to most of the existing and well-established numerical methods (finite volumes, finite elements). However, in the case of complex geometries, the cost for mesh generation might increase and become a severe constraint in complex fracture networks.

To solve the Darcy problem, we rely on different classes of numerical schemes: finite volumes, finite elements in primal and mixed formulations, and virtual elements in mixed formulation. For the finite volume class, we choose the two-point flux approximation (TPFA) on simplicial grids, applied to Problem 3; see [1] for details. This scheme is well known in the industry field and widely used for its velocity in assembling the discrete problem and for having a narrow matrix stencil. The scheme is locally conservative and robust with respect to strong variations of the effective hydraulic conductivity coefficient; however, it is consistent only for $K$-orthogonal grids. Regarding the class of finite elements, we consider mixed finite elements with the pair of spaces $\mathbb{R T}_{0}-\mathbb{P}_{0}$ for the Darcy velocity and piecewise constants for the hydraulic head, denoted by MFEM, defined on a simplicial grid; for further references, see [54, 55]. It is well known that $\mathbb{R} \mathbb{T}_{0}-\mathbb{P}_{0}$ is locally conservative and

Table 1 List of numerical schemes considered to solve the Darcy and heat problems, with the type of meshes used

\begin{tabular}{llll}
\hline Tag & Darcy eq. & Heat eq. & Grid \\
\hline TPFAUP & TPFA & TPFA + UPWIND & Matching triangles \\
MFEMUP & MFEM & TPFA + UPWIND & Matching triangles \\
MVEMUP & MVEM & TPFA + UPWIND & Matching polygons \\
MFEMSUPG & MFEM & FEM + SUPG & Matching triangles \\
FEMSUPG & OPT-FEM & OPT-FEM + SUPG & Non-match. triangles \\
XFEMSUPG & OPT-XFEM & OPT-XFEM + SUPG & Non-match. triangles \\
\hline
\end{tabular}




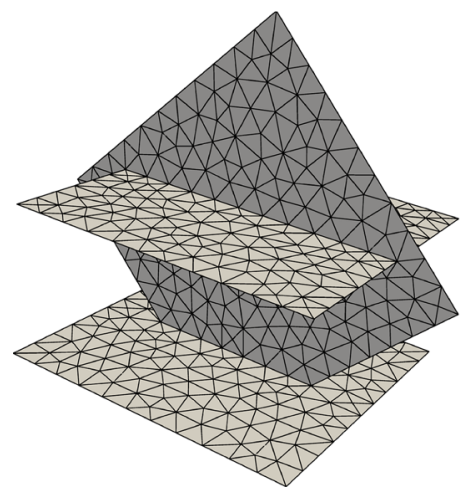

(a) matching triangles

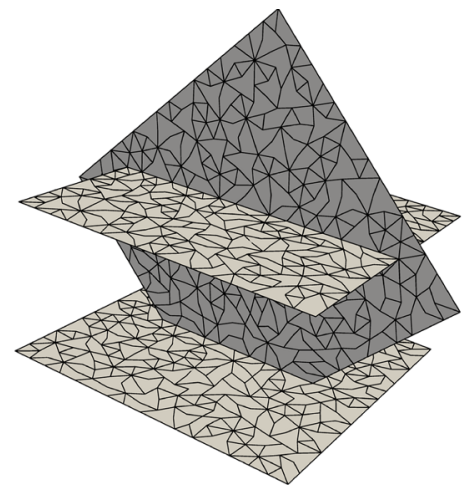

(b) matching polygons obtained by coarsening

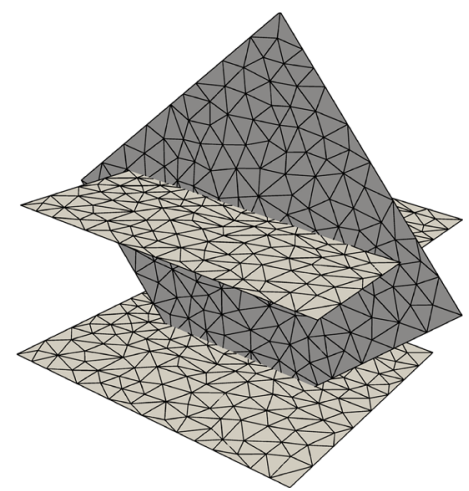

(c) non-matching triangles

Fig. 2 Examples of matching meshes

more robust than primal $\mathbb{P}_{1}$ finite elements with respect to strong variations on the effective hydraulic conductivity coefficient. On the other hand, it gives a larger linear system, with a saddle-point structure. The MFEM scheme solves the Darcy problem in the form presented in Problem 2. In some particular scenarios, the regularity requirements on meshes formed by triangles are too restrictive and schemes able to handle generally shaped cells are more suitable. In these cases, we rely on the new class of virtual element methods, which are variational methods where the basis functions of the discrete spaces are not prescribed a priori, and are defined implicitly on general star-shaped elements as solutions of suitable local PDEs. See the seminal works $[20,59,61,62]$ and those related to DFN [6-9, 34]. In our analysis, we consider only the lowest order mixed (MVEM) formulation, which can be viewed as a generalization $\mathbb{R T}_{0}-$ $\mathbb{P}_{0}$ mixed finite elements on generally shaped cells, solving Problem 2. Virtual element methods share many properties with their finite element counterpart: indeed, MVEM are locally conservative, robust with respect to the effective hydraulic conductivity variation and have the same grid stencil of the $\mathbb{R T}_{0}-\mathbb{P}_{0}$. Here, we use MVEM on polygonal grids, obtained by coarsening a mesh originally made of triangular elements in order to reduce the number of cells required in the simulation for complex geometries, as described in [33, 34].

To solve the heat equation (Problem 5), we use a TPFA scheme for the diffusive term and a weighted upwind scheme for the advective term. The advantage of this choice is that we obtain a stable scheme which respects the maximum and minimum principle, without oscillation due to high grid Péclet numbers. However, for some applications, the obtained scheme might be too diffusive. Finally, we consider also standard $\mathbb{P}_{1}$ finite elements, with a SUPG stabilized discrete variational formulation, which is a globally conservative numerical scheme.
Since in all the above cases we have matching meshes, and thus the degrees of freedom on the trace of one fracture correspond to the degrees of freedom of the intersecting one, coupling conditions can be imposed strongly.

Methods TPFAUP, MFEMUP, and MFEMSUPG are considered here standard reference approaches of different discretization strategies (finite volume and finite element based), and are used to benchmark the behavior of the other less conventional approaches, based e.g. on polygonal or non-matching mesh discretizations.

\subsection{Non-matching mesh discretization at traces}

When dealing with huge networks, the generation of conforming meshes may require a large computational cost. Then, it is worth considering a class of methods that do not require any kind of conformity of the fracture meshes to traces; see, for example, Fig. 2c.

In [11, 12, 14-17], a PDE-constrained optimization approach is proposed, based on non-conforming meshes, that can be applied both to Problems 3 and 5. In this framework, the problem is rewritten as a minimization problem for a functional measuring the error in fulfilling matching conditions, constrained by local PDEs on each fracture. This approach provides not only a numerical approximation of the solution but also a directly computed approximation of the flux exchanged at traces, which is of interest for many applications. The discretization can be based on different methods: standard $\mathbb{P}_{1}$ finite elements are the simplest choice, and the resulting scheme is denoted as OPT-FEM. However, as mesh elements arbitrarily cross the traces, the jump of the co-normal derivative of the solution at fracture intersections, still directly computed by the method, can not be correctly represented by nonconforming $\mathbb{P}_{1}$ finite elements. Thus, the use of local extended finite elements is also considered, being at the 
basis of the method denoted OPT-XFEM. When used for advection-dominated flow regimes, the SUPG-stabilized versions of the method are used (FEMSUPG, XFEMSUPG).

The resulting numerical schemes inherit the mass conservation properties of the local discrete formulations, thus they are globally but not locally conservative. A huge advantage of this method is that the matrices resulting from the local discrete problems can be computed in parallel, and also the solution can be computed strongly relying on parallel computing. In [19], a MPI-based parallel algorithm is proposed for assembling and solving the discrete problem using the conjugate gradient method on huge networks of fractures, whereas in [13] an implementation suitable for GPGPUs is presented.

\section{Examples}

In this section, we present three test cases with the aim of validating and comparing the previously introduced models. Extending the work proposed in [37], here we analyze the various approaches for time-dependent problems of advection-diffusion-reaction, where the advection velocity is computed by means of the same approach solving a diffusion problem. The key aspects of the various schemes will be highlighted and investigated, along with the impact of the lack of conservation of fluxes, both locally and through a trace, that characterizes some of the proposed approaches. For the considered test cases, both local and global quantities will be computed at different time steps, and used to assess and compare the behavior of the various approaches, such as (i) the integral mean in space of the temperature on a fracture $\Omega$, denoted as $\langle\theta\rangle_{\Omega}$; (ii) the total flux mismatch on a trace, $\delta \Phi_{\Gamma}$ defined as the integral of the sum of the net total fluxes $\Phi_{\Gamma, i}$ and $\Phi_{\Gamma, j}$ entering or leaving the two fractures $\Omega_{i}$ and $\Omega_{j}$ meeting at trace $\Gamma$, respectively, i.e., $\delta \Phi_{\Gamma}=\left|\Phi_{\Gamma, i}+\Phi_{\Gamma, j}\right|$ :

$\Phi_{\Gamma, i}=-\int_{\Gamma}\left(\llbracket \operatorname{tr}_{i} D_{i} \nabla \theta_{i} \cdot \boldsymbol{n}_{i \Gamma} \rrbracket_{\Gamma}+\llbracket \operatorname{tr}_{i} \theta_{i} \operatorname{tr}_{i} \boldsymbol{u}_{i} \cdot \boldsymbol{n}_{i \Gamma} \rrbracket_{\Gamma}\right)$

with $\boldsymbol{n}_{i}$ the unit normal vectors to $\Gamma$, with fixed orientation on $\Omega_{i}$; and (iii) the averaged $\theta$ on the outflow boundary, $\partial \Omega_{D}^{\text {outflow }}$, denoted as:

$\langle\theta\rangle_{\text {outflow }}=\frac{1}{\left|\partial \Omega_{D}^{\text {outflow }}\right|} \int_{\partial \Omega_{D}^{\text {outflow }}} \operatorname{tr} \theta$.

Furthermore, we denote the norm of total flux at each trace $\Gamma$ on $\Omega_{i}$ as:

$\hat{\Phi}_{\Gamma, i}(t)=\left\|\llbracket \operatorname{tr}_{i} D_{i} \nabla \theta_{i} \cdot \boldsymbol{n}_{i} \rrbracket_{\Gamma}+\llbracket \operatorname{tr}_{i} \theta_{i} \operatorname{tr}_{i} \boldsymbol{u}_{\boldsymbol{i}} \cdot \boldsymbol{n}_{i} \rrbracket_{\Gamma}\right\|$.

The considered test cases are designed in order to challenge the proposed approaches with complex geometries and/or realistic models. In Section 4.1, we consider the effect of a vanishing trace from a simple network, by a sequence of simulations. In the second example presented in Section 4.2, a synthetic small network of 10 fractures is considered, to analyze the behavior of the methods on a more general yet simple configuration. We finally conclude with a realistic example in Section 4.3, where a network of 89 fractures is generated from an extrusion of an interpreted natural outcrop and physically sound values for the various parameters are used.

With the purpose of establishing a standard reference for the analysis of numerical schemes for flow in fractures, test cases in Sections 4.1 and 4.3 are borrowed from [37] and adapted to the present context, all the geometrical data being available in [36].

\subsection{Vanishing trace between intersecting fractures}

As a first test case, named test case 1, the same setting of the problem proposed in the example of [37, Subsection 4.3.1] is considered. In the reference, the Darcy problem was tackled, whereas here a non-stationary advection-diffusion problem for the passive scalar $\theta$ is solved.

In this test case, the same problem is solved on different geometries. Let us consider a network composed of three fractures, named $\Omega_{l}, \Omega_{r}$, and $\Omega_{c}$, as shown in Fig. 3 . Fracture $\Omega_{l}$ has a fixed position, whereas fractures $\Omega_{r}$ and $\Omega_{c}$ are displaced, for each different geometry of a same distance along the $z$-direction, such that the length of the intersection line between fractures $\Omega_{l}$ and $\Omega_{r}$, denoted as $\Gamma_{0}$, progressively reduces from the configuration shown in Fig. 5, being instead fixed at the intersection between $\Omega_{r}$ and $\Omega_{c}$, denoted as $\Gamma_{1}$. In such a way, 21 different configurations are obtained, with the length of the vanishing trace $\Gamma_{0}$ ranging from 0.6 at configuration

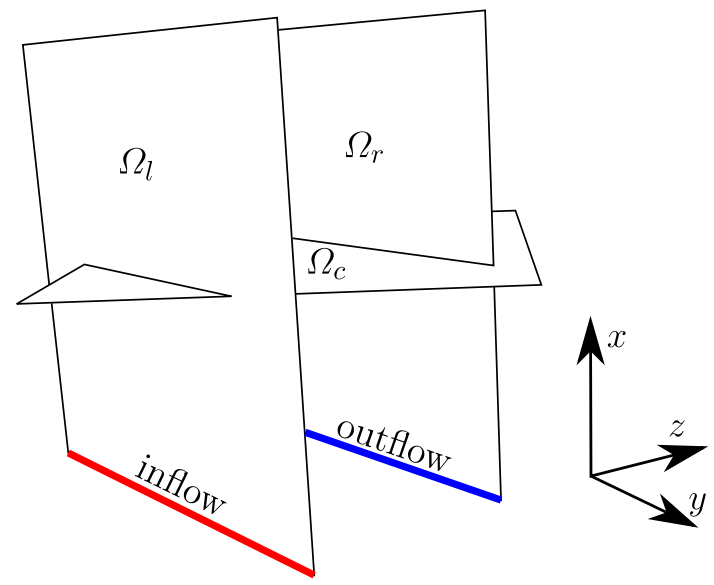

Fig. 3 Geometry of test case 1. The inflow and outflow portions of the boundary are represented by red and blue lines, respectively 
$C 0$, as shown in Fig. 3, to 0.01 , at configuration $C 20$. The geometries corresponding to configurations $C 0, C 10$, and $C 20$ are shown in Fig. 5. For each configuration, the Darcy problem, formulated as in (2) or (3), depending on the method, is first solved in order to compute the Darcy velocity $\boldsymbol{u}$, with a null source term. A unitary effective hydraulic conductivity $K$ is used for all the fractures and pressure head boundary conditions are prescribed on the bottom part of $\partial \Omega_{l}$ (inflow) and $\partial \Omega_{r}$ (outflow) equal to 1 and 0 , respectively (see Fig. 3). On the other portions of the boundary, a no-flux boundary condition is imposed. Subsequently, an advection-diffusion problem is solved, obtained from Problem 5 setting the reaction operator $r(\cdot, \cdot) \equiv 0$, and with null source. We assume a unitary coefficient $\zeta$, a diffusion coefficient $D$ equal to $10^{-4}$, and a constant in time unitary Dirichlet boundary condition on the inflow part of the domain boundary, whereas homogeneous Neumann boundary conditions are set on the rest of the boundary.

The meshes used for the space discretization are different for conforming-triangular, conforming-polygonal, and nonmatching gridding strategies, as discussed above. The mesh for the conforming mesh schemes is generated using the Gmsh [40] library, and two different mesh parameters are used, corresponding to about $10^{3}$ and $10^{4}$ elements for the configuration $C 0$. Clearly, as the length of trace $\Gamma_{0}$ progressively reduces from configuration $C 0$ to $C 20$, the mesh generation tool tends to increase the number of elements in order to meet the conformity requirement without compromising mesh quality. This process inevitably leads to a large increment of the number of elements from configuration $C 0$ to $C 20$, for each of the three initial refinement levels. The mesh for the non-matching mesh schemes is obtained through the Triangle [57] software, using two mesh parameters, again corresponding to about $10^{3}$ and $10^{4}$ elements. In this case, instead, since the mesh is independent from the traces, the number of elements is practically unaffected by the change of the geometry from configurations $C 0$ to $C 20$ (small oscillations of few elements are observed as a consequence of the change of the coordinates of fracture $\Omega_{l}$ ). The polygonal mesh for the MVEM approach is finally built gluing together the triangular elements of the conforming mesh, thus aiming at mitigating the increase of mesh elements and degrees of freedom. The number of elements for the various schemes, for each geometrical configuration, is reported in Fig. 4, on the leftmost column, for the coarse (top) and fine (bottom) meshes. The center and right columns of Fig. 4 report instead the number of degrees of freedom corresponding to each method, which can be used as an indication of the corresponding computational cost. We remark, however, that the computational cost is also largely affected by the approach used to solve linear systems: some of the proposed methods, e.g., have efficient parallel solvers,
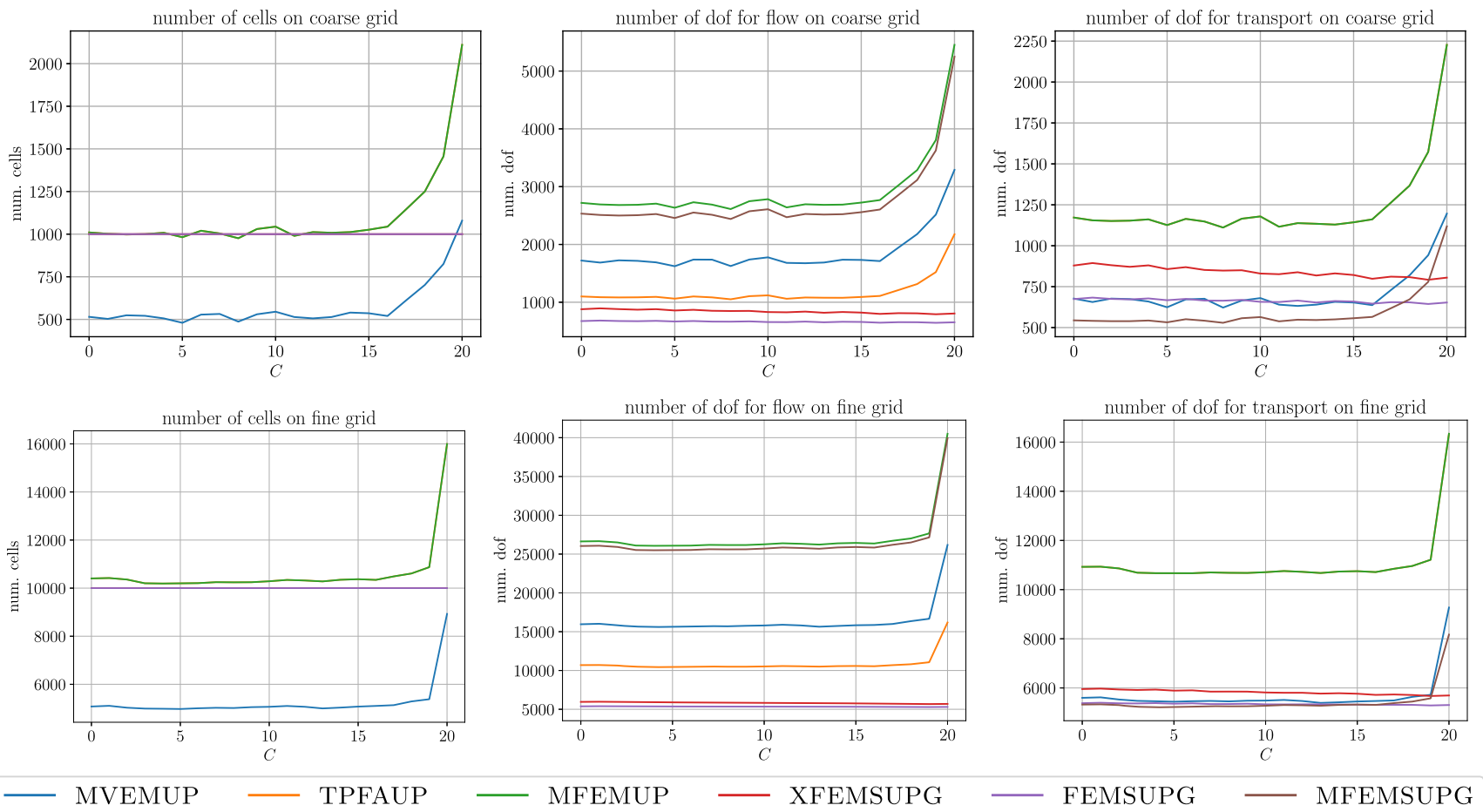

Fig. 4 Number of cells (left), number of DOFs for the Darcy problem (middle), and number of DOFs for the dispersion problem (right) against configuration id for the two mesh parameters of test case 1, coarse mesh on the top and fine mesh on the bottom grids 

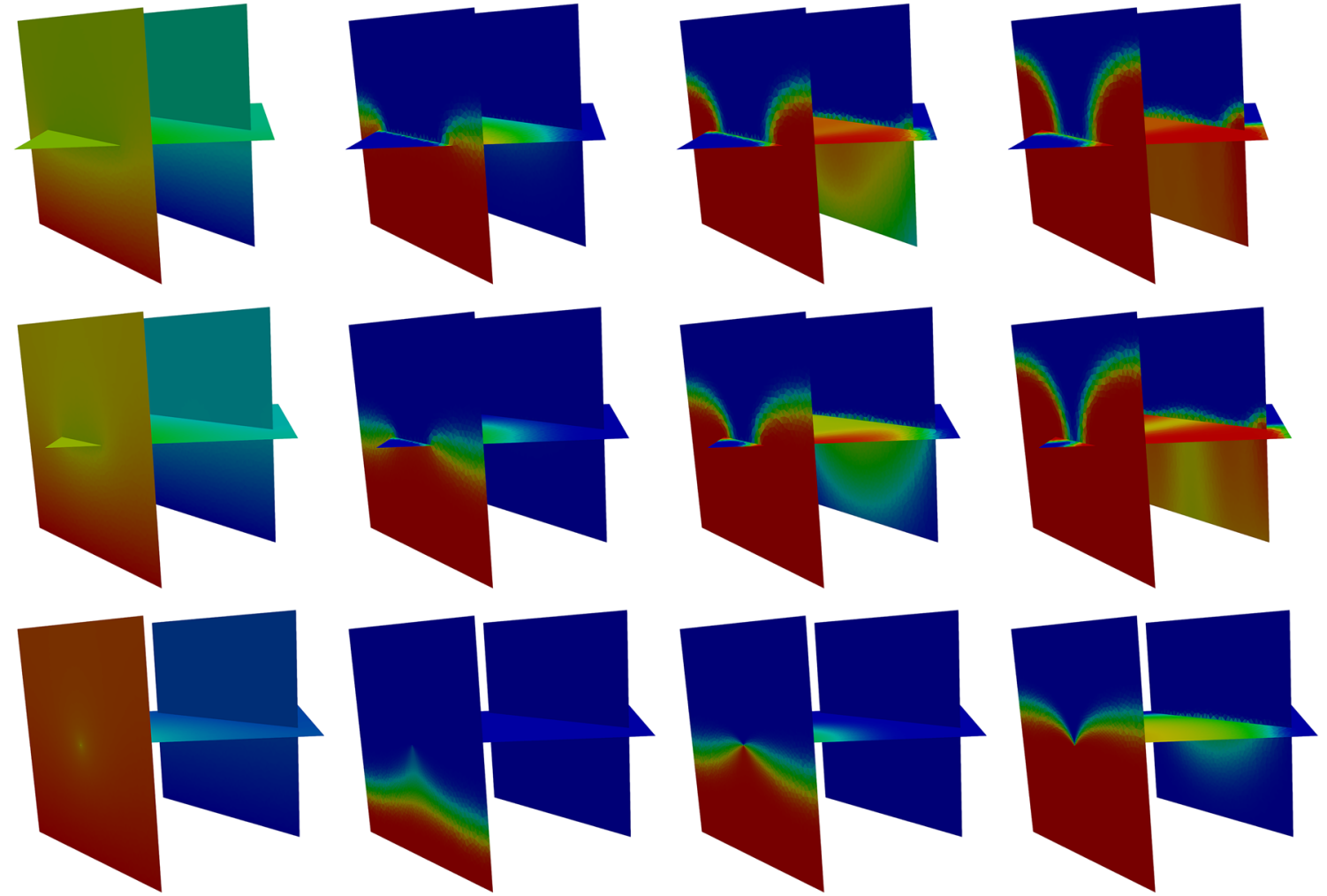

Fig. 5 Solution of test case 1 obtained with the MFEMUP scheme. On the pressure head solution is shown on the leftmost column at configurations $C 0, C 10$, and $C 20$. On the remaining columns, the solution $\theta$ is represented at $t=\{1.25,2.5,5\}$, respectively. The color scale spans from 0 to 1

others take advantage of standard domain decomposition strategies to achieve computational efficiency. Also, the availability of effective preconditioners should be taken into account. Here, however, the main focus is on the analysis of the response of the methods in terms of prediction accuracy versus geometrical and model complexities typical of DFN simulations, thus we refer to the literature of each method for further details on computational efficiency issues.

In the following of this test case, for brevity, the mesh with $10^{3}$ cells on configuration $C 0$ will be denoted as coarse mesh and the mesh with $10^{4}$ cells on $C 0$ as the fine. For time discretization an equally spaced mesh with time-step equal to 0.05 is considered and 300 time-steps are performed, starting from an all zero initial condition.

The solution obtained with the scheme MFEMUP is reported, as an example, in Fig. 5 on configurations $C 0$, $C 10$, and $C 20$. On the leftmost column, the pressure head distribution in the network is shown for the three geometries, whereas the remaining columns depict the temperature distribution $\theta$ at three time-steps corresponding to a time $t=1.25, t=2.5$, and $t=5$, respectively. We can notice that, as the trace between fractures $\Omega_{l}$ and $\Omega_{r}$ vanishes, the pressure head distribution displays a steeper gradient around the intersection, and the effective permeability of the network reduces, thus also reducing the
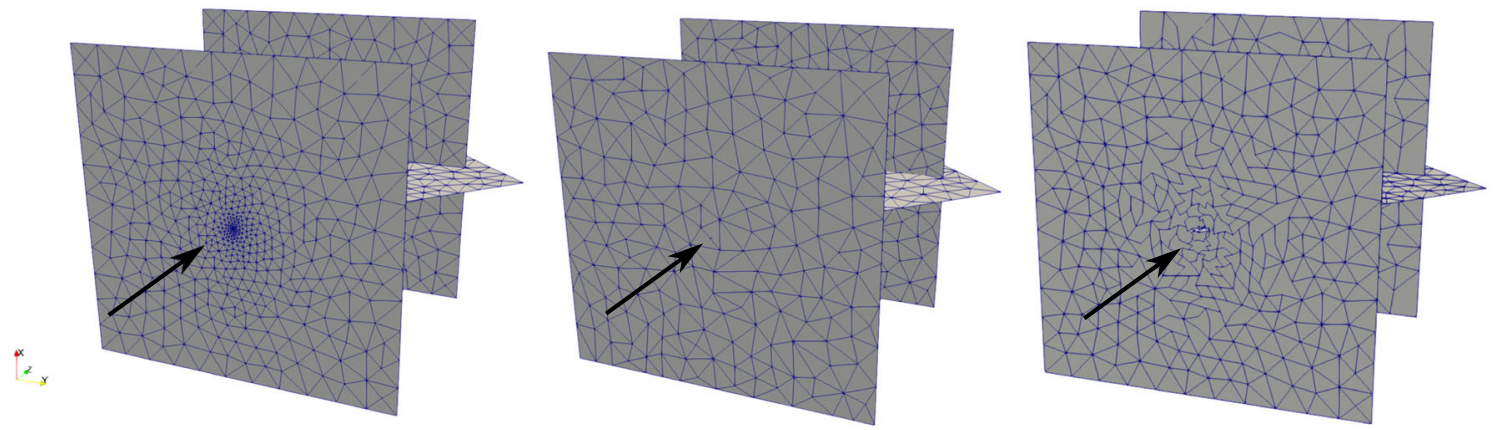

Fig. 6 Mesh for test case 1 on configuration $C 20$ for conforming (left) non-conforming (middle) and coarsened (right) schemes 

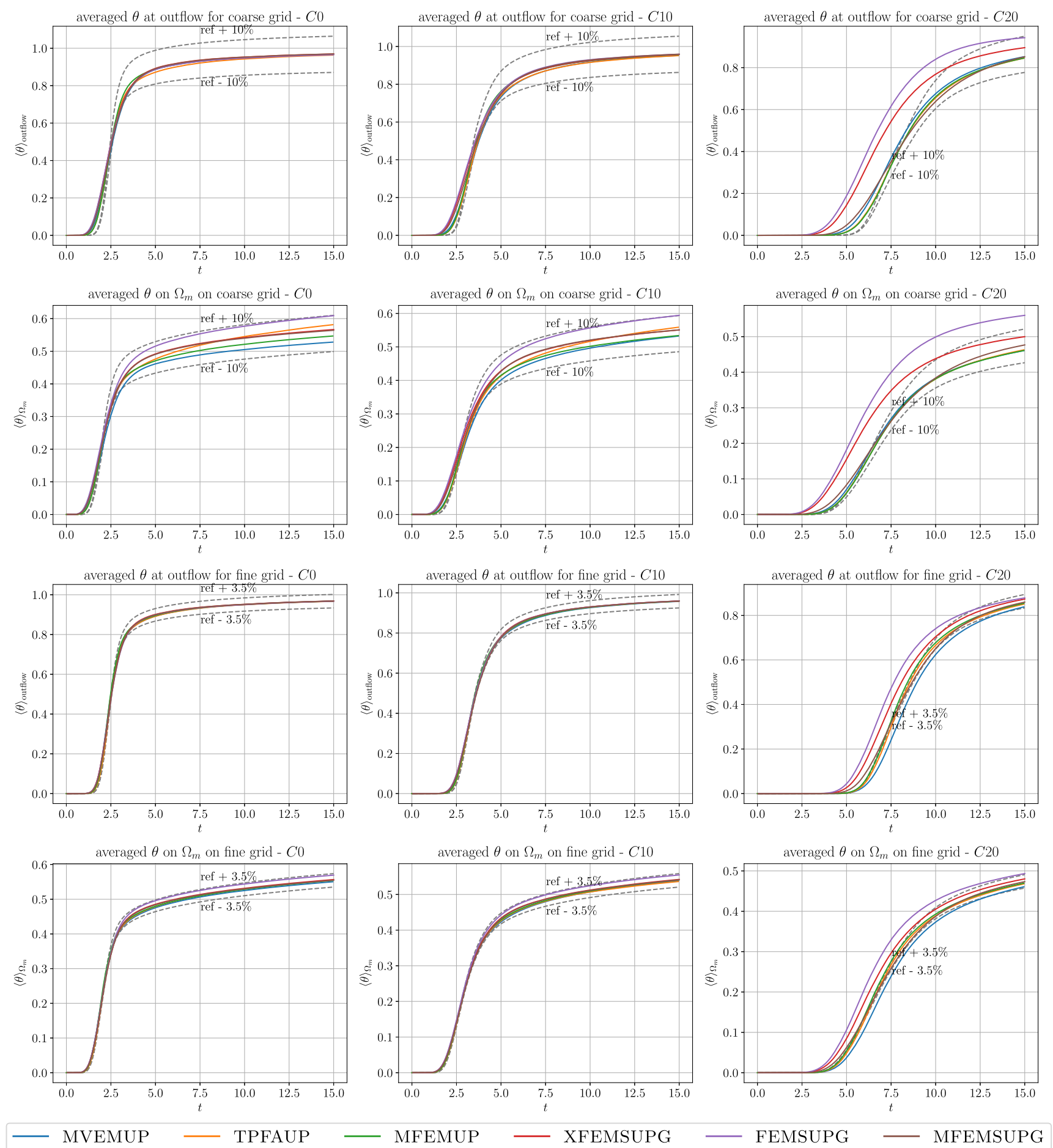

Fig. 7 Average temperature on curves against time for test case 1 on the coarsest (first six pictures) and finest (last six pictures) for selected fracture and configurations. Columns refer to the same geometrical configuration: $C 0$ on column $1, C 10$ on column 2 , and $C 20$ on column 3 . The average temperature curves refer to fracture $\Omega_{m}$ penetration depth of the higher temperature zone in the network at fixed time frames. From a computational point of view, simulations become more and more challenging as the solution starts to display steep gradients, especially for methods built on non-conforming meshes, that are not adapted to the geometry, as shown in Fig. 6.

For all the proposed numerical schemes, at each timestep, the following quantities are computed: the average 

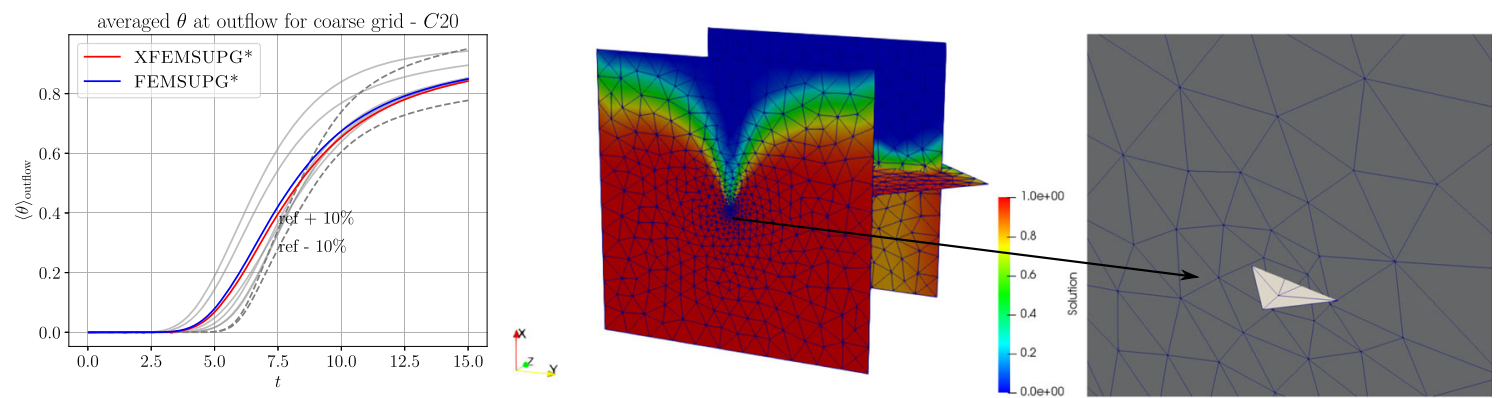

Fig. 8 Outflow average temperature curve with XFEMSUPG and FEMSUPG approaches (left), and solution with XFEMSUPG at $t=15$ with a zoom of the mesh around $\Gamma_{0}$ (right), both on the perturbed mesh of configuration $C 20$

temperature $\langle\theta\rangle_{\Omega}$, the average temperature $\langle\theta\rangle_{\text {outflow }}$ on the outflow portion of the boundary, and, for non-locally conservative schemes, as the optimization-based methods, the total flux mismatch $\delta \Phi_{\Gamma}$ at each trace.

A reference solution is computed using the MFEMUP method on a mesh much finer than the three considered here for the simulations, counting about $6.3 \times 10^{5}$ cells, almost independently from the configuration id, as cell size is capable of resolving the smallest length of the vanishing trace.

The plots in Fig. 7 propose a comparison of average temperature for all the proposed methods against time, on three selected configurations, for all the considered meshes. In the picture, dashed black lines represent relative errors with respect to the reference solution. As expected, all the curves are in very good agreement, also on the coarsest mesh, for configuration $C 0$, whereas small discrepancies appear for configuration $C 10$ on the coarse mesh that however disappear as the mesh is refined. Larger differences appear, for methods FEMSUPG and XFEMSUPG, instead for the simulations on configuration $C 20$. This is expected, since, as mentioned, when the varying trace becomes very small, methods built on non-matching meshes can not rely on the effect of mesh refinement around the vanishing trace which clearly improves representation capabilities of conforming methods. We observe that method MVEMUP retains good approximation capabilities despite the coarsening.

In order to quantify what is the effect of mesh adaptation due to conformity requirements on the quality of the solution with respect to the effect of the approximations introduced by the non-matching schemes themselves, in
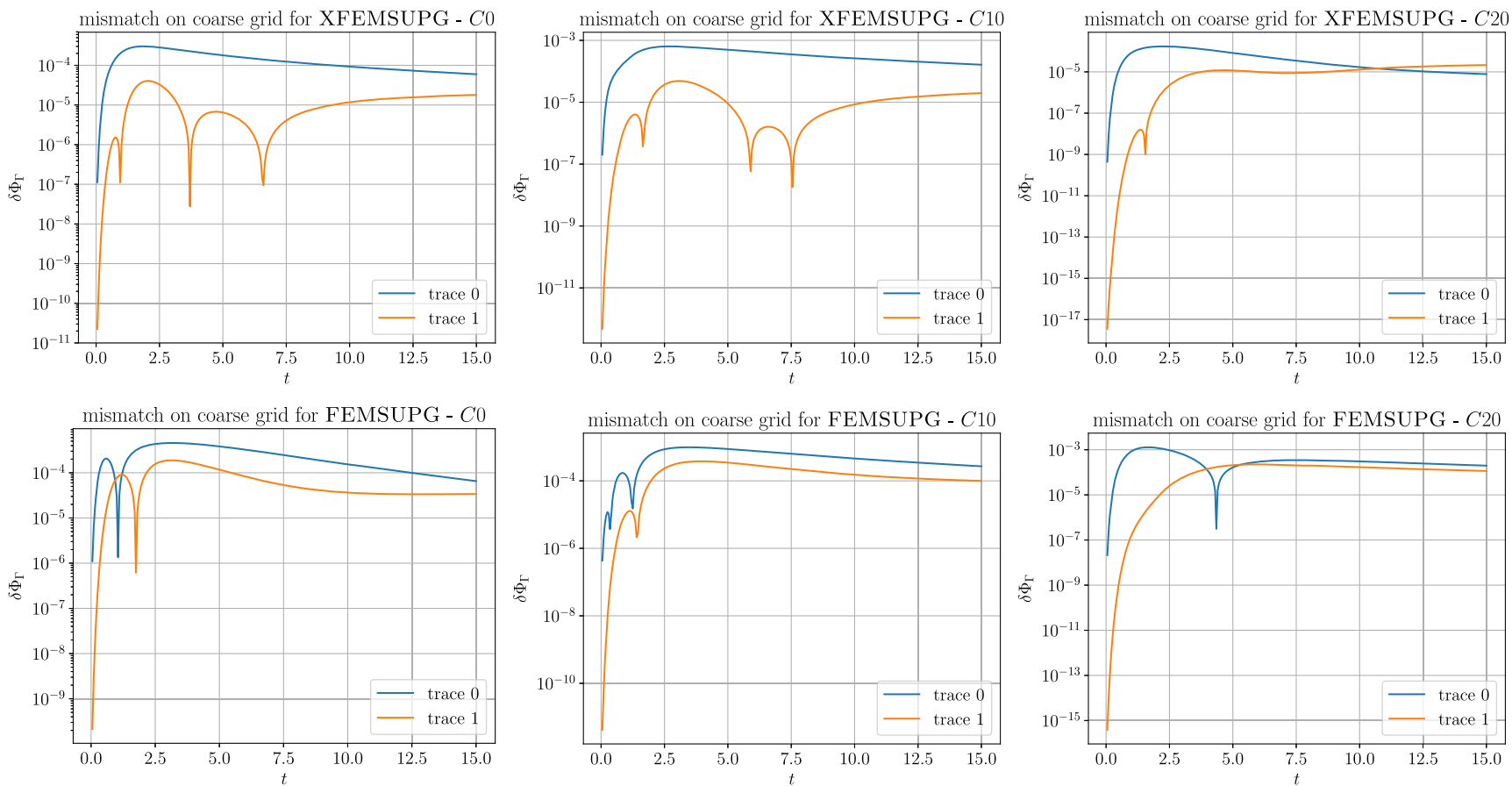

Fig. 9 Total flux mismatch against time for test case 1 on traces $\Gamma_{0}$ (top) and $\Gamma_{1}$ (bottom) for configuration $C 0$ (left), $C 10$ (middle), and $C 20$ (right) 
Table 2 Maximum value in time of total flux norms $\hat{\Phi}_{\Gamma_{0}}$ and $\hat{\Phi}_{\Gamma_{1}}$ on traces $\Gamma_{0}$ and $\Gamma_{1}$ of test case 1 , respectively, computed with the XFEMSUPG method on configurations $C 0, C 10$, and $C 20$

\begin{tabular}{llll}
\hline & $C 0$ & $C 10$ & $C 20$ \\
\hline$\hat{\Phi}_{\Gamma_{0}}$ & 0.6072 & 0.4725 & 0.251 \\
$\hat{\Phi}_{\Gamma_{1}}$ & 0.876 & 0.912 & 1.300 \\
\hline
\end{tabular}

Fig. 8 (left) the outflow average temperature for the methods XFEMSUPG and FEMSUPG is reported against time, for configuration $C 20$ on a perturbation of the coarse mesh used for the conforming methods for configuration $C 20$, overlapped to the curves of the other schemes, in transparency, on the original meshes. We observe that the curves of methods FEMSUPG and XFEMSUPG on the perturbed adapted mesh are now much closer to the curves of other conforming approaches, thus clearly showing the effect of mesh adaptation on the quality of the solution. The solution obtained with the XFEMSUPG approach on the perturbed mesh at $t=15$ is reported in Fig. 8, on the right, along with a detail of the mesh around $\Gamma_{0}$, clearly showing the non conformity of the mesh. Two aspects are to be remarked: first, the non-conforming approaches are capable of producing reasonable approximations of the solution also on the coarse mesh, which can be greatly improved refining the mesh and still using a fraction of the degrees of freedom required by the conforming approaches, see, e.g., the last two plots in the third column of Fig. 7; second, nonconforming-mesh approaches allow to freely choose the refinement level of the mesh, thus allowing to efficiently use mesh adaptation strategies, only refining the mesh where required, independently of the geometrical constraints [12].

As the non-conforming mesh approaches XFEMSUPG and FEMSUPG are non locally conservative, Fig. 9 reports the value of $\delta \Phi_{\Gamma}$ against time on the coarsest mesh for the two traces of this example at configurations $C 0, C 10$, and $C 20$, from left to right, respectively, results with the XFEMSUPG approach are on the top, results with FEMSUPG on the bottom. The maximum-in-time total flux norms on traces $\Gamma_{0}$ and $\Gamma_{1}$ are reported in Table 2, computed on the finest mesh as $\hat{\Phi}_{\Gamma_{0}}=\max _{t} \frac{1}{2}\left(\hat{\Phi}_{\Gamma_{0}, l}+\hat{\Phi}_{\Gamma_{0}, c}\right)$, and $\hat{\Phi}_{\Gamma_{1}}=\max _{t} \frac{1}{2}\left(\hat{\Phi}_{\Gamma_{1}, c}+\hat{\Phi}_{\Gamma_{1}, r}\right)$, used to compute relative flux mismatches. We can see that relative values of less than $1 \%$ are obtained for all times for both methods, with the higher values corresponding to the configuration $C 20$, as expected. Moreover, for the larger times, mismatch values tend to decrease or to remain constant at a fixed value. Thus, this non-local conservation has a negligible impact on the computed solution. Furthermore, mismatch errors can be reduced by refining the mesh.
The mesh Péclet number for this problem ranges between a maximum of about $6 \times 10^{2}$ to a minimum of about 100 on the computational meshes for XFEMSUPG, FEMSUPG, and MFEMSUPG methods, thus a streamline upwind PetrovGalerkin (SUPG) stabilization strategy was adopted. As a consequence, small overshots/undershots in the solution are observed in SUPG-stabilized methods, as well known in the literature, whereas the intrinsic diffusive behavior of methods using upwinding for advection prevents this kind of phenomena.

\subsection{Synthetic network}

In the second test, labeled test case 2 , we consider a more complex network composed of 10 fractures with 14 traces, thus being more similar to (a portion of) realistic DFNs, still remaining simple enough to perform analyses on the solutions obtained with the considered schemes. The network is represented in Fig. 10, where the inflow and outflow portions of the boundary are also marked. Also, in this case, the Darcy velocity $\boldsymbol{u}$ is first computed solving Problem 2 or 3, depending on the chosen numerical scheme, with constant effective hydraulic conductivity equal to 1 on all fractures and null source term. A unitary pressure head drop is imposed between the inflow and outflow portions of the border, all other fracture edges being insulated. The Darcy velocity is then used as an input for an advectiondiffusion problem for a scalar quantity $\theta$, as formulated in (5), with null source and reaction terms. A coefficient $\zeta=1$ is chosen, whereas the diffusion coefficient is $D=10^{-4}$. A unitary Dirichlet boundary condition is prescribed on the inflow border and all other edges are insulated.

Two meshes are used also for test case 2 counting about $10^{3}$ elements (coarse mesh) and $4 \times 10^{4}$ elements (fine mesh), respectively. The mesh Péclet number, related to the coarse mesh for SUPG-stabilized methods, is of about 100 . An equally spaced mesh is then used for time discretization with 500 steps of length 0.05 . Also, in this case, the initial solution is the null function. An example of the obtained numerical solution with the TPFA method is shown in Fig. 11: in the first column on the left, the pressure head distribution in the network is represented, solution of the Darcy problem on the coarse (top) and fine (bottom) meshes; the remaining columns depict the solution $\theta$ of the dispersion problem at three selected time frames, corresponding to $t=3.35, t=6.25$, and $t=12.5$, again on the coarse (top) and fine (bottom) meshes. Coherently, the heat flows from the inflow to the outflow by following a tortuous path given by the complex disposition of the fractures and traces. The solution on the coarse grid has a spreader front than on the fine grid due to the artificial diffusivity of the scheme. 


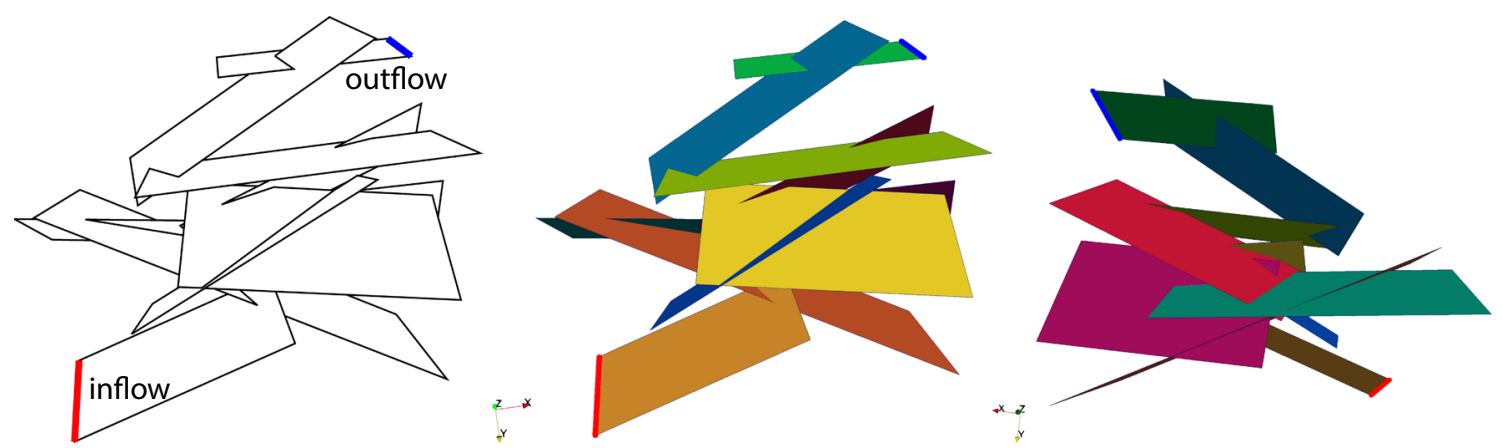

Fig. 10 Geometry of test case 2 and two views of the network. A red line represents the inflow and a blue thick line the outflow part of the boundary, respectively

As previously, the average temperature $\langle\theta\rangle_{\Omega}$ on selected fractures, the average outflow temperature $\langle\theta\rangle_{\text {outflow }}$, and flux mismatch $\delta \Phi_{\Gamma}$ at traces are used to compare and assess the approximation capabilities of the various schemes. The curves of $\langle\theta\rangle_{\Omega_{1}},\langle\theta\rangle_{\Omega_{3}}$, and $\langle\theta\rangle_{\text {outflow }}$ are reported in Fig. 12. A reference solution is computed with the MFEMSUPG method on a mesh counting about $2 \times 10^{4}$ cells, and relative error curves with respect to this solution are shown in dashed lines in Fig. 12. We can observe that, despite the network having a larger number of fractures and fracture intersections with respect to test case 1 , all the methods, in the absence of severe geometrical features have good approximation properties that further improve with mesh refinement. The larger differences are observed for the average outflow temperature curve related to the MVEMUP approach on the coarse mesh and for the average temperature curves of FEMSUPG again on the coarse mesh. In both cases, differences slightly exceed $10 \%$ of the reference, and are reduced by mesh refinement. Concerning the MVEMUP, the difference is caused by the coarsening process which reduces the number of elements of the original mesh to about one-half. Also, the TPFA method used for advection is observed to have poor performances on irregular polygonal cells, as the ones generated by the coarsening method. Concerning the FEMSUPG method, some discrepancies with respect to the reference are to be expected, as the approach is designed to be computationally inexpensive; nonetheless, it is capable of providing satisfactory predictions.

Curves of the total flux mismatch at the traces are reported against time in Fig. 13 for the FEMSUPG and XFEMSUPG methods. In this picture, values of $\delta \Phi_{\Gamma}$ are shown, without labels, for all the traces in the network, highlighting that, in all cases, the errors remain limited in time. Furthermore, maximum-in-time mismatch values are lower than $1 \%$ of the maximum in time total flux norm, for

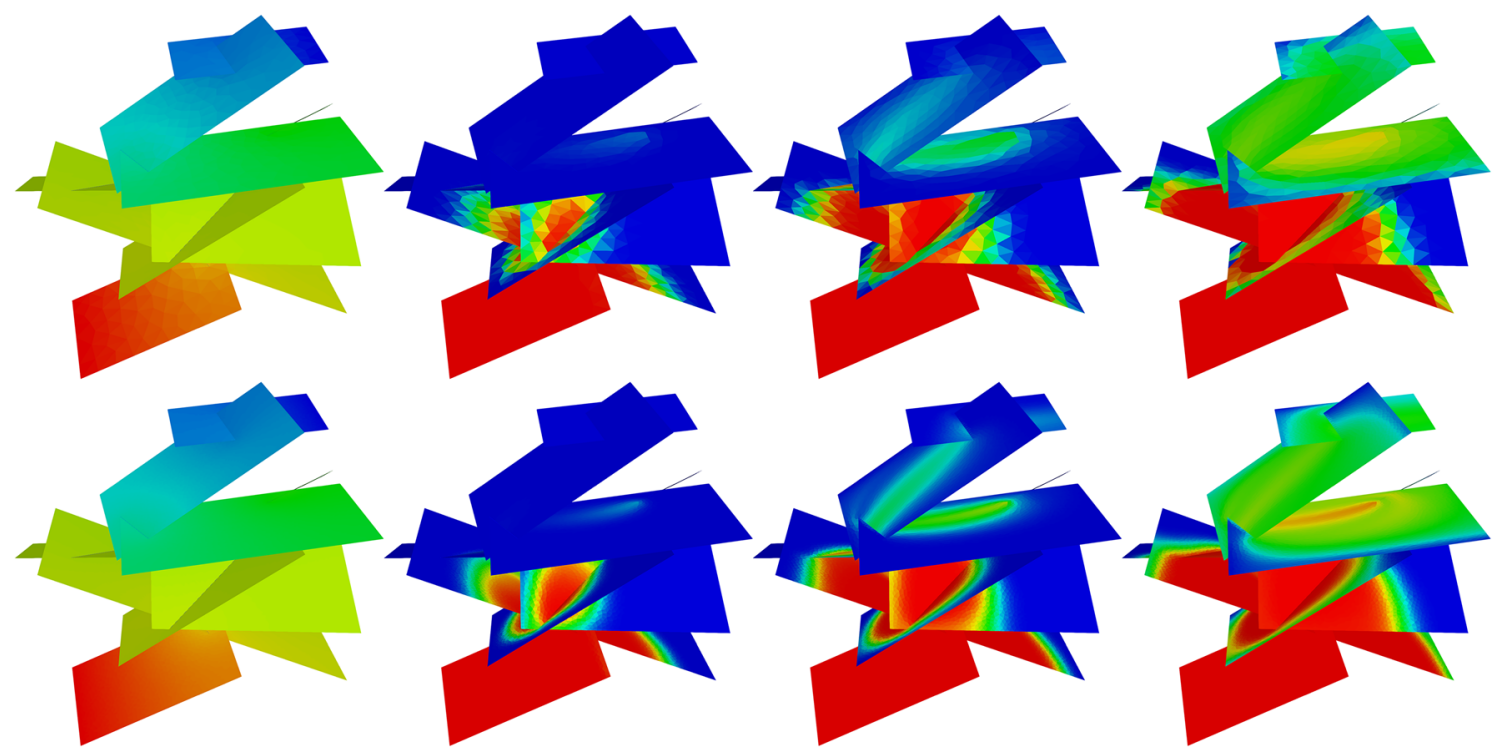

Fig. 11 Solution of the test case in Section 4.2. On the first column, the pressure head solution for the two grid refinements. The other columns show $\theta$ at different times of $3.35,6.25$, and 12.5 , respectively, for both levels of refinement. In all cases, the solution is rescaled in the range [0,1] 

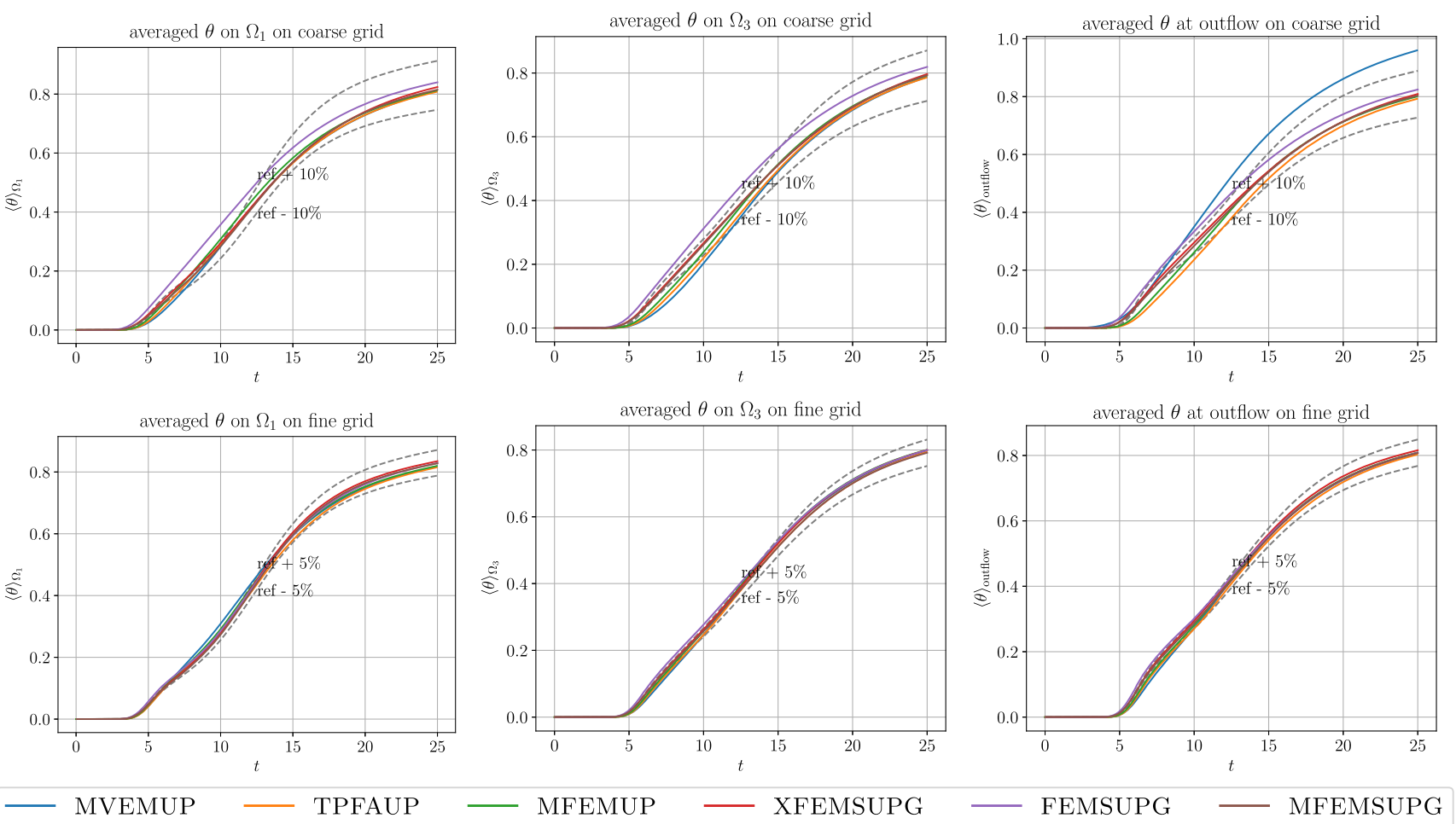

Fig. 12 Average temperature on two selected fractures (left and middle columns) and at outflow (right column) curves against time for test case 2. Coarse mesh on top, fine mesh at the bottom

all the traces; values of the maximum flux norm with respect to time on each trace are reported in Table 3, computed with the XFEMSUPG method on the finest mesh.

\subsection{Extruded real outcrop}

In this last test case, we consider a fracture network generated from an extruded outcrop, located in Western Norway. The test case is inspired by Section 4.4 of [37]. The network is composed by $N_{\Omega}=89$ intersecting fractures resulting in 166 traces. There are 7 non-connected fractures and with no flow boundary conditions, which will not contribute to the solution. The geometry is depicted in Fig. 14. The aim of this test case is to validate the proposed numerical schemes in the presence of realistic physical parameters of a real fracture network. However, we assume that all the fractures share the same values of effective hydraulic conductivity and heat diffusion coefficient.

We consider two distinct problems that differ from each other from the position of the inflow and outflow
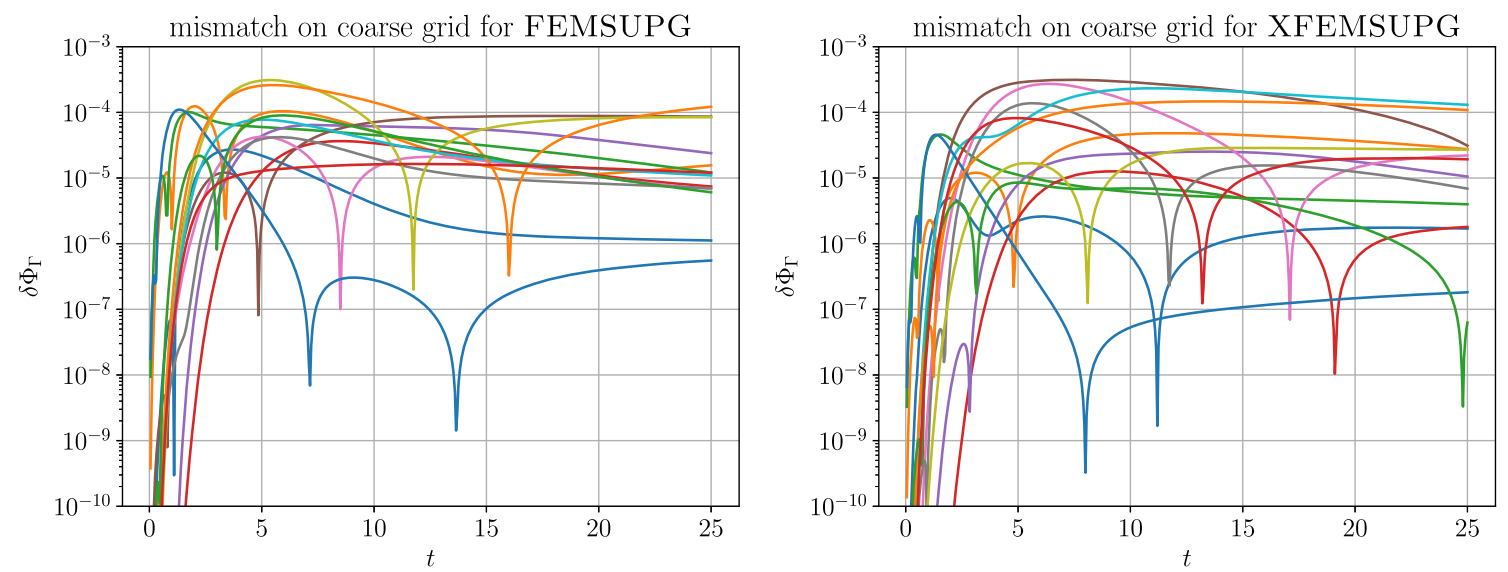

Fig. 13 Total flux mismatch against time for all the traces of the network of test case 2 on the coarse mesh with methods FEMSUPG (left) and XFEMSUPG (right) 
Table 3 Maximum value in time of total flux norm across each trace of the DFN for test case 2 computed with the XFEMSUPG method on the fine mesh

\begin{tabular}{cllllll}
\hline$\hat{\Phi}_{\Gamma_{1}}$ & $\hat{\Phi}_{\Gamma_{3}}$ & $\hat{\Phi}_{\Gamma_{4}}$ & $\hat{\Phi}_{\Gamma_{5}}$ & $\hat{\Phi}_{\Gamma_{6}}$ & $\hat{\Phi}_{\Gamma_{7}}$ \\
0.040 & $\hat{\Phi}_{\Gamma_{2}}$ & 0.150 & 0.260 & 0.284 & 0.047 & 0.074 \\
$\hat{\Phi}_{\Gamma_{8}}$ & 0.079 & $\hat{\Phi}_{\Gamma_{10}}$ & $\hat{\Phi}_{\Gamma_{11}}$ & $\hat{\Phi}_{\Gamma_{12}}$ & $\hat{\Phi}_{\Gamma_{13}}$ & $\hat{\Phi}_{\Gamma_{14}}$ \\
0.020 & $\hat{\Phi}_{\Gamma_{9}}$ & 0.163 & 0.443 & 0.061 & 0.058 & 0.063 \\
\hline
\end{tabular}

boundaries. In the first case (denoted as case 1), the inflow and outflow are imposed on two different fractures, while in the second case (case 2) they belong to the same fracture. See Fig. 14 where a sketch of the network is shown with the position of the inflow and outflow boundaries. On the other portions of the boundaries, a no flow boundary condition is given. In both cases, we require a simulation grid with roughly $70 \mathrm{k}$ elements.

Fractures are immersed in granite and we assume that at the beginning of the simulation we have thermal equilibrium, the water contained in the fractures is at $\overline{\bar{\theta}}_{i}=$ $353.15 \mathrm{~K}\left(80{ }^{\circ} \mathrm{C}\right)$, for all $i=1, \ldots, N_{\Omega}$. The value of $\hat{\theta}_{i}$, which represents the constant temperature of the rock matrix, is at the same value, $\hat{\theta}_{i}=\overline{\bar{\theta}}_{i}$, for all $i=1, \ldots, N_{\Omega}$. The relations to compute the physical parameters for the simulations are the ones presented in Section 2.2. We assume $\epsilon_{i}=2 \mathrm{~mm} \forall i$, and $\phi_{i}=0.95 \forall i$. The water and rock physical parameters are reported in Table 4 .

From these data, we obtain: $\forall i=1, \ldots, N_{\Omega}$,

$$
\begin{aligned}
K_{i} & \approx 1.84 \times 10^{-6} \mathrm{~m}^{2} / \mathrm{s}, & c_{e, i} & \approx 4,000,700 \mathrm{~J} /\left(\mathrm{m}^{3} \mathrm{~K}\right), \\
\lambda_{e, i} & \approx 0.72 \mathrm{~W} /(\mathrm{m} \mathrm{K}), & \zeta_{i} & \approx 1.95 \times 10^{-3} \mathrm{~m}, \\
D_{i} & \approx 0.35 \times 10^{-9} \mathrm{~m}^{3} / \mathrm{s}, & \iota_{i} & \approx 3.05 \times 10^{-10} \mathrm{~m} / \mathrm{s} .
\end{aligned}
$$

Regarding boundary conditions, for the Darcy problem, we impose a pressure head equal to $\bar{h}_{\text {inflow }}=2.5 \mathrm{~km}$ at the inflow boundary and $\bar{h}_{\text {outflow }}=0 \mathrm{~m}$ at the outflow

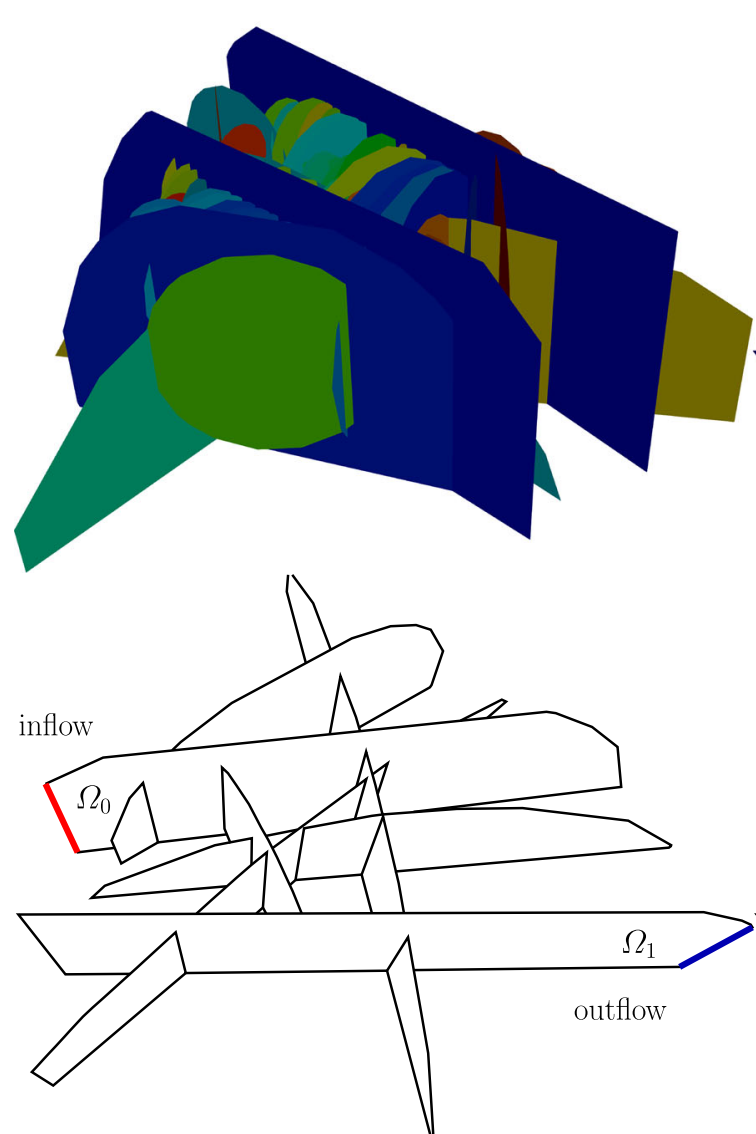

Fig. 14 Geometry of the test case in Section 4.3. On the top, two screenshots of the geometry of the network. On the bottom, a sketch of the network with only the largest fractures to present the boundary
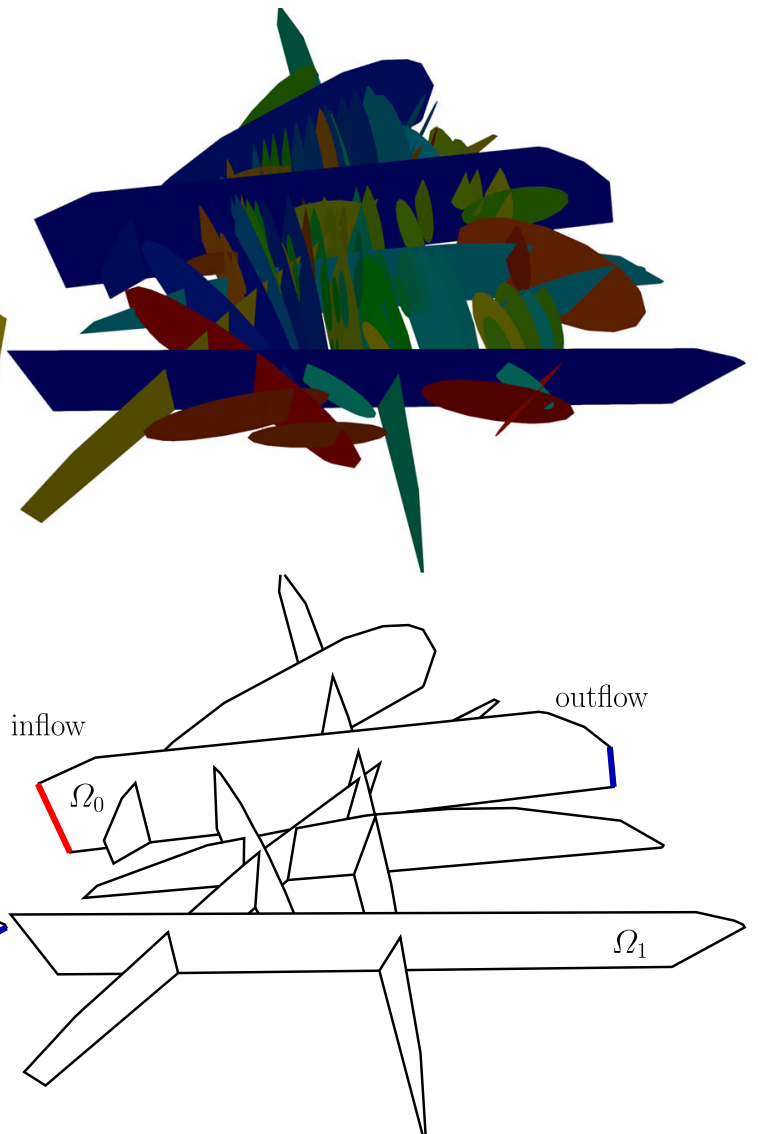

conditions: on the left in the case of inflow and outflow imposed on two different fractures, on the right on the same fracture. The inflow is represented in red and the outflow in blue 
Table 4 List of rock and water coefficients for the example in Section 4.3

\begin{tabular}{lll}
\hline & Water & Rock \\
\hline Dynamic viscosity & $\mu=3.55 \mathrm{P} \mathrm{a} \mathrm{s}$ & - \\
Thermal conductivity & $\lambda_{w}=0.667 \mathrm{~W} /(\mathrm{m} \mathrm{K})$ & $\lambda_{m}=3.07 \mathrm{~W} /(\mathrm{m} \mathrm{K})$ \\
Density & $\rho_{w}=1000 \mathrm{~kg} / \mathrm{m}^{3}$ & $\rho_{m}=2700 \mathrm{~kg} / \mathrm{m}^{3}$ \\
Specific heat capacity & $c_{w}=4099 \mathrm{~J} /(\mathrm{kg} \mathrm{K})$ & $c_{m}=790 \mathrm{~J} /(\mathrm{kg} \mathrm{K})$ \\
Heat transfer coefficient & $\gamma=1.25 \times 10^{-3} \mathrm{~W} /\left(\mathrm{m}^{2} \mathrm{~K}\right)$ & \\
\hline
\end{tabular}
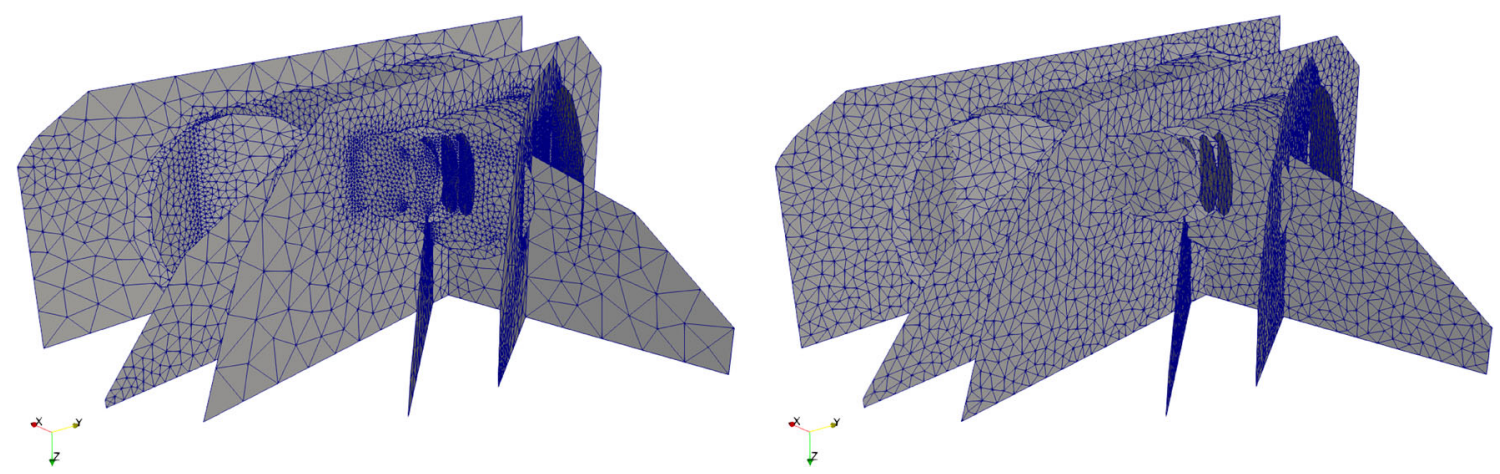

Fig. 15 Conforming (left) and non-matching (right) meshes for test case 3

Fig. 16 Solution with the MFEMUP scheme for the test case 3: case 1 on the top; case 2 at the bottom. The first column shows the pressure head solution, the second column the temperature distribution at the end of time evolution. On the left, the fractures depicted in blue are unconnected to the main network. In this case, we set their pressure at zero. On the left, the temperature of these fractures stays constant during the simulation

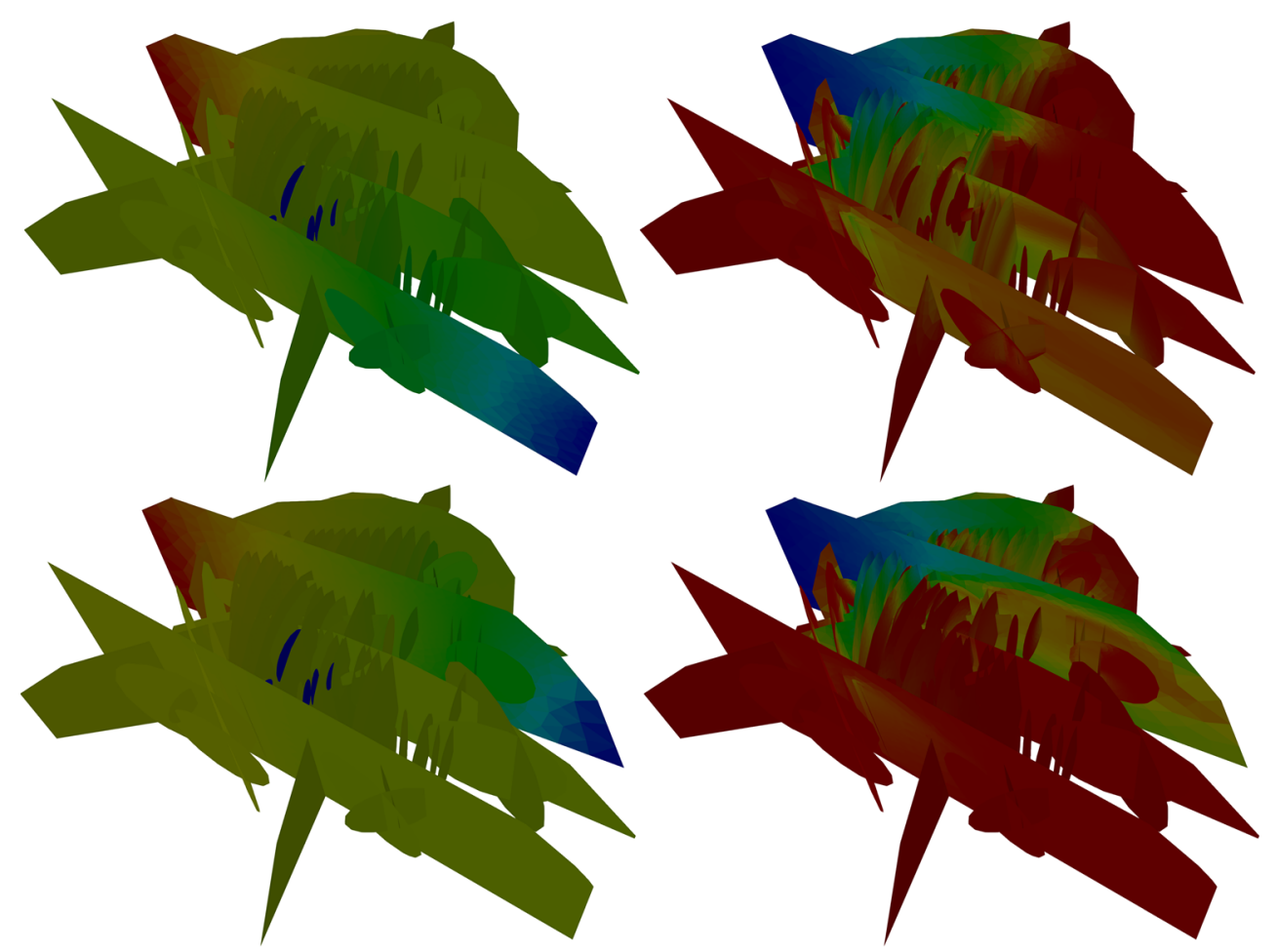



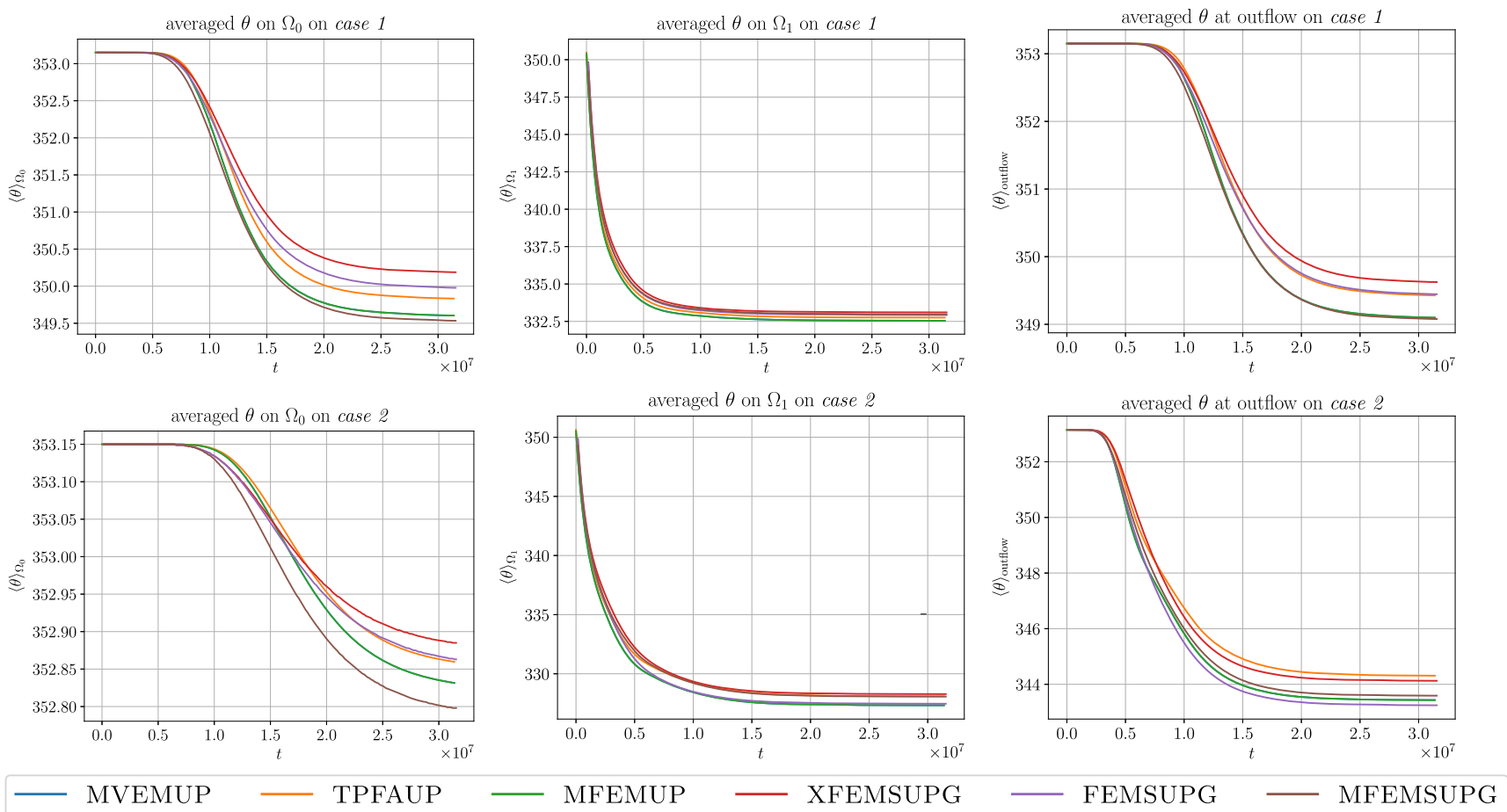

Fig. 17 Curves of average theta on fractures $\Omega_{0}$ (left) and $\Omega_{1}$ and at outflow (right) against time for test case 3: case 1 top, case 2 bottom

boundary, while for the heat problem we impose $\bar{\theta}_{\text {inflow }}=$ $303.15 \mathrm{~K}\left(30{ }^{\circ} \mathrm{C}\right)$ at the inflow and zero diffusive flux at the outflow. The simulation time is a year $\left(3.154 \times 10^{7} \mathrm{~s}\right)$, divided in 200 time-steps.

The conforming computational mesh counts about $7 \times$ $10^{4}$ elements, while the non-matching computational mesh has $2 \times 10^{4}$ cells and is shown in Fig. 15. It is possible to notice how, in order to meet the conformity requirement, mesh elements of the conforming mesh are concentrated near the traces, whereas the non-matching mesh has all elements of equal size evenly distributed in the network. The non-matching mesh is characterized by a mesh Péclet number of about $3 \times 10^{4}$ for case 1 and $6.6 \times 10^{4}$ for case 2 , reached on fracture $\Omega_{0}$, in both cases. The nonadapted mesh and the high mesh Péclet numbers make this example extremely complex for methods built on non-conforming meshes and relying on stabilization for advection-dominated flow regimes.

The computed solution with the MFEMUP scheme is reported in Fig. 16 where we display the solution of the Darcy problem on the left and the solution at the end of time evolution on the right, for both the setting of case 1, on top, and case 2, at the bottom.

Figure 17 shows the curves against time of the average temperature on the inflow $\left(\Omega_{0}\right)$ and outflow $\left(\Omega_{1}\right)$ fractures, along with the average temperature on the outflow boundary. Despite the complexity of the geometry and of the model, curves appear in good agreement. For this last example, no coarsening was used for the MVEMUP method, as the poor performances of the TPFA method on polygonal cells, already observed in test case 2 , have a strong impact on the quality of the solution in this more complex case. The curves related to the MVEMUP approach on triangular meshes are almost perfectly overlapped to the curves of the MFEMUP method. Curves of XFEMSUPG and FEMSUPG are in good agreement with those of the other methods. Flux mismatch values have been computed for these methods for those traces having a non-negligible maximum-in-time total flux norm, i.e., traces $\Gamma_{k}$ with $\hat{\Phi}_{\Gamma_{k}}$ greater than $1 \%$ of $\max _{k} \hat{\Phi}_{\Gamma_{k}}$, i.e., the maximum total flux norm through all the traces in the network. Indeed, in this more complex example, some traces are excluded from the main flow path, and thus have an almost zero net flux. The computed relative mismatch values are lower than $1 \%$ also for this example.

\section{Conclusions}

In this work, we presented a detailed comparative study of several solution strategies for single-phase flow and transport in discrete fracture networks. The proposed numerical schemes are challenged with networks of increasing geometrical complexity and with unsteady advectionreaction-diffusion problems. The characteristics of the 
various approaches are compared in terms of flexibility in handling geometrical complexity, local and global conservativity, and stability to high Péclet numbers. The main focus of the present work is the response of the considered numerical schemes to geometrical complexity and thus other relevant aspects such as the impact of different time integration schemes, or of strongly heterogeneous fracture properties, such as fracture transmissivity, and also a more detailed discussion on computational cost, have not been addressed.

Methods based on matching grids at the traces trade simplicity in imposing coupling conditions with the lack of control on the number of mesh elements, which is actually constrained by the conformity requirement. On the other hand, non-matching and polygonal-based approaches demand ad hoc discretization strategies but allow full flexibility in meshing. The obtained results show that the presence of traces much smaller than the characteristic size of the network is a major source of complexity. Methods based on non-matching meshes and polygonal meshes are both capable of producing reliable approximations, also in time-evolving simulations. Lack of local conservation at traces appears to have an acceptable impact on the quality of the solution and on the measured global quantities, compared with other sources of error and to the usual uncertainty in model parameters. This is a non-trivial result, mainly for time-evolving simulations, where an accumulation of local errors could have a strong impact on the solution. Peclét-related instabilities are effectively cured by SUPG stabilization, providing solutions comparable with those obtained by methods intrinsically stable thanks to numerical diffusivity.

Non-matching mesh discretizations are thus suggested to provide reliable predictions in complex geometrical configurations, and benefit from mesh adaptivity to recover high accuracy levels. The VEM-based approach based on the coarsening of a conforming mesh allows to mitigate the impact of handling large meshes for complex geometries, and is promising of being effective on complex geometrical configurations in combination with more advanced discretization strategies for unstationary advection-reaction simulations.

Acknowledgements Open access funding provided by Politecnico di Milano within the CRUI-CARE Agreement. The authors warmly thank Luisa F. Zuluaga for constructing and providing the real fracture network for the example in Section 4.3. Finally, the authors warmly thank the PorePy development team for many fruitful discussions related to the development of this work.

Funding This research has been partially supported by the MIUR project "Dipartimenti di Eccellenza 2018 - 2022" (CUP E11G18000350001), PRIN project "Virtual Element Methods: Analysis and Applications" (201744KLJL_004) and by INdAM - GNCS. The second author received financial support for the ANIGMA project from the Research Council of Norway (project no. 244129/E20) through the ENERGIX program and from the "Visiting Professor Project" of Politecnico di Torino. The first and third author received financial support from Politecnico di Torino through project "Starting grant RTD."

Open Access This article is licensed under a Creative Commons Attribution 4.0 International License, which permits use, sharing, adaptation, distribution and reproduction in any medium or format, as long as you give appropriate credit to the original author(s) and the source, provide a link to the Creative Commons licence, and indicate if changes were made. The images or other third party material in this article are included in the article's Creative Commons licence, unless indicated otherwise in a credit line to the material. If material is not included in the article's Creative Commons licence and your intended use is not permitted by statutory regulation or exceeds the permitted use, you will need to obtain permission directly from the copyright holder. To view a copy of this licence, visit http:// creativecommonshorg/licenses/by/4.0/.

\section{References}

1. Aavatsmark, I.: Interpretation of a two-point flux stencil for skew parallelogram grids. Comput. Geosci. 11(3), 199-206 (2007). https://doi.org/10.1007/s10596-007-9042-1

2. Antonietti, P.F., Facciolà, C., Russo, A., Verani, M.: Discontinuous galerkin approximation of flows in fractured porous media on polytopic grids. SIAM J. Sci. Comput. 41(1), A109-A138 (2019). https://doi.org/10.1137/17M1138194

3. Antonietti, P.F., Formaggia, L., Scotti, A., Verani, M., Verzotti, N.: Mimetic finite difference approximation of flows in fractured porous media. ESAIM:, M2AN 50(3), 809-832 (2016). https://doi.org/10.1051/m2an/2015087

4. Bear, J.: Dynamics of Fluids in Porous Media. American Elsevier, New York (1972)

5. Benedetto, M., Berrone, S., Borio, A., Pieraccini, S., Scialò, S.: Order preserving SUPG stabilization for the virtual element formulation of advection-diffusion problems. Comput. Methods Appl. Mech. Eng. 311, 18-40 (2016). https://doi.org/10.1016/j. cma.2016.07.043

6. Benedetto, M.F., Berrone, S., Borio, A., Pieraccini, S., Scialò, S.: A hybrid mortar virtual element method for discrete fracture network simulations. J. Comput. Phys. 306, 148-166 (2016). https:// doi.org/10.1016/j.jcp.2015.11.034. http://www.sciencedirect.com/ science/article/pii/S0021999115007743

7. Benedetto, M.F., Berrone, S., Pieraccini, S., Scialò, S.: The virtual element method for discrete fracture network simulations. Comput. Methods Appl. Mech. Eng. 280(0), 135-156 (2014). https://doi.org/10.1016/j.cma.2014.07.016. http://www. sciencedirect.com/science/article/pii/S0045782514002485

8. Benedetto, M.F., Berrone, S., Scialò, S.: A globally conforming method for solving flow in discrete fracture networks using the virtual element method. Finite Elem. Anal. Des. 109, 23-36 (2016). https://doi.org/10.1016/j.finel.2015.10.003

9. Benedetto, M.F., Borio, A., Scialò, S.: Mixed virtual elements for discrete fracture network simulations. Finite Elem. Anal. Des. 134, 55-67 (2017). https://doi.org/10.1016/j.finel.2017.05.011. http://www.sciencedirect.com/science/article/pii/ S0168874X17302500

10. Berrone, S., Borio, A.: Orthogonal polynomials in badly shaped polygonal elements for the Virtual Element Method. Finite Elem. Anal. Des. 129, 14-31 (2017). https://doi.org/10.1016/j.finel. 2017.01.006 
11. Berrone, S., Borio, A., Scialò, S.: A posteriori error estimate for a PDE-constrained optimization formulation for the flow in DFNs. SIAM J. Numer. Anal. 54(1), 242-261 (2016). https://doi.org/10. $1137 / 15 \mathrm{M} 1014760$

12. Berrone, S., Borio, A., Vicini, F.: Reliable a posteriori mesh adaptivity in discrete fracture network flow simulations. Comput. Meth. Appl. Mech. Eng. 354, 904-931 (2019). https://doi.org/10. 1016/j.cma.2019.06.007

13. Berrone, S., D'Auria, A., Vicini, F.: Fast and robust flow simulations in discrete fracture networks with GPGPUs. GEM Int. J. Geomath. 10(1), 8 (2019)

14. Berrone, S., Pieraccini, S., Scialò, S.: A PDE-constrained optimization formulation for discrete fracture network flows. SIAM J. Sci. Comput. 35(2), B487-B510 (2013). https://doi.org/10.1137/ 120865884

15. Berrone, S., Pieraccini, S., Scialò, S.: On simulations of discrete fracture network flows with an optimization-based extended finite element method. SIAM J. Sci. Comput. 35(2), 908-935 (2013). 10.1137/120882883

16. Berrone, S., Pieraccini, S., Scialò, S.: An optimization approach for large scale simulations of discrete fracture network flows. J. Comput. Phys. 256, 838-853 (2014). https://doi.org/10.1016/j.jcp. 2013.09.028

17. Berrone, S., Pieraccini, S., Scialò, S.: Towards effective flow simulations in realistic discrete fracture networks. J. Comput. Phys. 310, 181-201 (2016). https://doi.org/10.1016/j.jcp.2016.01.009

18. Berrone, S., Pieraccini, S., Scialò, S.: Flow simulations in porous media with immersed intersecting fractures. J. Comput. Phys. 345, 768-791 (2017). https://doi.org/10.1016/j.jcp.2017.05.049

19. Berrone, S., Scialò, S., Vicini, F.: Parallel meshing, discretization and computation of flow in massive Discrete Fracture Networks. SIAM J. Sci. Comput. 41(4), C317-C338 (2019). https://doi.org/ 10.1137/18M1228736

20. Brezzi, F., Falk, R.S., Marini, D.L.: Basic principles of mixed virtual element methods. ESAIM Math. Model. Numer. Anal. 48(4), 1227-1240 (2014). https://doi.org/10.1051/m2an/2013138

21. Burman, E., Hansbo, P., Larson, M.G., Larsson, K.: Cut finite elements for convection in fractured domains. Comput. Fluids 179, 726-734 (2019). https://doi.org/10.1016/j.compfluid.2018.07.022

22. Chave, F., Di Pietro, D.A., Formaggia, L.: A hybrid high-order method for darcy flows in fractured porous media. SIAM J. Sci. Comput. 40(2), A1063-A1094 (2018). https://doi.org/10.1137/17 M1119500

23. Chave, F., Di Pietro, D.A., Formaggia, L.: A hybrid high-order method for passive transport in fractured porous media. GEM - Int J Geomath 10(1), 12 (2019). https://doi.org/10.1007/s13137-0190114-x

24. D'Angelo, C., Scotti, A.: A mixed finite element method for Darcy flow in fractured porous media with non-matching grids. Math. Model. Numer. Anal. 46(02), 465-489 (2012). https://doi.org/10. $1051 / \mathrm{m} 2 \mathrm{an} / 2011148$

25. Dowd, P.A., Martin, J.A., Xu, C., Fowell, R.J., Mardia, K.V.: A three-dimensional fracture network data set for a block of granite. Int. J. Rock Mech. Min. Sci. 46(5), 811-818 (2009). https://doi. org/10.1016/j.ijrmms.2009.02.001

26. de Dreuzy, J.R., Méheust, Y., Pichot, G.: Influence of fracture scale heterogeneity on the flow properties of three-dimensional discrete fracture networks (dfn). J. Geophys. Res. Solid Earth 117(B11). https://doi.org/10.1029/2012JB009461. https:// agupubs.onlinelibrary.wiley.com/doi/abs/10.1029/2012JB009461 (2012)

27. de Dreuzy, J.R., Pichot, G., Poirriez, B., Erhel, J.: Synthetic benchmark for modeling flow in $3 \mathrm{~d}$ fractured media. Comput Geosci 50, 59-71 (2013). https://doi.org/10.1016/j.cageo.2012.07.025. http:// www.sciencedirect.com/science/article/pii/S0098300412002683.
Benchmark problems, datasets and methodologies for the computational geosciences

28. Erhel, J., de Dreuzy, J.R., Poirriez, B.: Flow simulation in threedimensional discrete fracture networks. SIAM J. Sci. Comput. 31(4), 2688-2705 (2009). https://doi.org/10.1137/080729244

29. Facciolà, C., Antonietti, P.F., Verani, M.: Mixed-primal discontinuous galerkin approximation of flows in fractured porous media on polygonal and polyhedral grids. PAMM 19(1), e201900117 (2019). https://doi.org/10.1002/pamm.201900117. https:// onlinelibrary.wiley.com/doi/abs/10.1002/pamm.201900117

30. Flemisch, B., Berre, I., Boon, W., Fumagalli, A., Schwenck, N., Scotti, A., Stefansson, I., Tatomir, A.: Benchmarks for singlephase flow in fractured porous media. Adv. Water Resour. 111, 239-258 (2018). https://doi.org/10.1016/j.advwatres.2017. 10.036. https://www.sciencedirect.com/science/article/pii/S03091 70817300143

31. Formaggia, L., Fumagalli, A., Scotti, A., Ruffo, P.: A reduced model for Darcy's problem in networks of fractures. Esaim Math. Model. Numer. Anal. 48, 1089-1116 (2014). https://doi.org/10.1051/m2an/2013132. https://www.esaim-m2an. org/articles/m2an/abs/2014/04/m2an130132/m2an130132.html

32. Franca, L.P., Frey, S.L., Hughes, T.: Stabilized finite element methods: I. Application to the advective-diffusive model. Comput. Methods Appl. Mech. Eng. 95(2), 253-276 (1992)

33. Fumagalli, A.: Dual virtual element method in presence of an inclusion. Appl. Math. Lett. 86, 22-29 (2018). https://doi.org/10. 1016/j.aml.2018.06.004. https://www.sciencedirect.com/science/ article/pii/S0893965918301812

34. Fumagalli, A., Keilegavlen, E.: Dual virtual element method for discrete fractures networks. SIAM J. Sci. Comput. 40(1), B228B258 (2018). https://doi.org/10.1137/16M1098231. https://epubs. siam.org/doi/abs/10.1137/16M1098231

35. Fumagalli, A., Keilegavlen, E.: Dual virtual element methods for discrete fracture matrix models. Oil Gas Sci. Technol. 74(41), 1-17 (2019). https://doi.org/10.2516/ogst/2019008. https://ogst.ifpenergiesnouvelles.fr/articles/ogst/full_html/2019/ 01/ogst170210/ogst170210.html

36. Fumagalli, A., Keilegavlen, E., Scialò, S.: Input and benchmarking data for flow simulations in discrete fracture networks. Data Brief 21, 1135-1139 (2018). https://doi.org/10.1016/j.dib.2018.10.088

37. Fumagalli, A., Keilegavlen, E., Scialò, S.: Conforming, nonconforming and non-matching discretization couplings in discrete fracture network simulations. J. Comput. Phys. 376, 694-712 (2019). https://doi.org/10.1016/j.jcp.2018.09.048. https://www. sciencedirect.com/science/article/pii/S0021999118306508

38. Fumagalli, A., Scotti, A.: A numerical method for two-phase flow in fractured porous media with non-matching grids. Adv. Water Resour. 62 Part C(0), 454-464 (2013). https://doi.org/10.1016/j. advwatres.2013.04.001. https://www.sciencedirect.com/science/ article/pii/S0309170813000523. Computational Methods in Geologic CO2 Sequestration

39. Fumagalli, A., Scotti, A.: A Reduced Model for Flow and Transport in Fractured Porous Media with Non-Matching Grids, pp. 499-507. Springer, Berlin (2013). https://link.springer.com/ chapter/10.1007/978-3-642-33134-3_53

40. Geuzaine, C., Remacle, J.F.: Gmsh: a 3-d finite element mesh generator with built-in pre- and post-processing facilities. Int. J. Numer. Methods Eng. 79(11), 1309-1331 (2009). https://doi.org/ $10.1002 / \mathrm{nme} .2579$

41. Hardebol, N.J., Maier, C., Nick, H., Geiger, S., Bertotti, G., Boro, H.: Multiscale fracture network characterization and impact on flow: a case study on the latemar carbonate platform. J. Geophys. Res. Solid Earth 120(12), 8197-8222 (2015). https://doi.org/10. 1002/2015JB011879. 2015JB011879 
42. Hyman, J.D., Gable, C.W., Painter, S.L., Makedonska, N.: Conforming delaunay triangulation of stochastically generated three dimensional discrete fracture networks: a feature rejection algorithm for meshing strategy. SIAM J. Sci. Comput. 36(4), A1871-A1894 (2014). https://doi.org/10.1137/130942541. http:// epubs.siam.org/doi/abs/10.1137/130942541

43. Hyman, J.D., Karra, S., Makedonska, N., Gable, C.W., Painter, S.L., Viswanathan, H.S.: Dfnworks: a discrete fracture network framework for modeling subsurface flow and transport. Comput. Geosci. 84, 10-19 (2015). https://doi.org/10.1016/j.cageo.2015. 08.001. http://linkinghub.elsevier.com/retrieve/pii/S00983004153 0026110.1016/j.cageo.2015.08.001

44. Keilegavlen, E., Berge, R., Fumagalli, A., Starnoni, M., Stefansson, I., Varela, J., Berre, I.: Porepy: an open-source software for simulation of multiphysics processes in fractured porous media. Tech. rep., arXiv:1908.09869 [math.NA] (2019)

45. Keilegavlen, E., Fumagalli, A., Berge, R., Stefansson, I.: Implementation of mixed-dimensional models for flow in fractured porous media. In: Radu, F.A., Kumar, K., Berre, I., Nordbotten, J.M., Pop, I.S. (eds.) Numerical Mathematics and Advanced Applications ENUMATH 2017, vol. 126, pp. 573-580. Springer International Publishing (2019). https://doi.org/10.1007/978-3319-96415-7_52

46. Lee, S., Lee, Y.J., Wheeler, M.F.: A locally conservative enriched galerkin approximation and efficient solver for elliptic and parabolic problems. SIAM J. Sci. Comput. 38(3), A1404-A1429 (2016). https://doi.org/10.1137/15M1041109

47. Martin, V., Jaffré, J., Roberts, J.E.: Modeling fractures and barriers as interfaces for flow in porous media. SIAM J. Sci. Comput. 26(5), 1667-1691 (2005). https://doi.org/10.1137/ S1064827503429363. http://scitation.aip.org/getabs/servlet/ GetabsServlet?prog=normal\&id=SJOCE3000026000005001667 000001\&idtype $=$ cvips\&gifs $=$ yes

48. McClure, M., Babazadeh, M., Shiozawa, S., Huang, J.: Fully coupled hydromechanical simulation of hydraulic fracturing in threedimensional discrete fracture networks. In: SPE Hydraulic Fracturing Technology Conference. Society of Petroleum Engineers (2015). https://doi.org/10.2118/173354-MS. http://www.onepetro. org/doi/10.2118/173354-MS

49. Mustapha, H., Mustapha, K.: A new approach to simulating flow in discrete fracture networks with an optimized mesh. SIAM J. Sci. Comput. 29(4), 1439-1459 (2007). https://doi.org/10.1137/06 0653482

50. Ngo, T.D., Fourno, A., Noetinger, B.: Modeling of transport processes through large-scale discrete fracture networks using conforming meshes and open-source software. J. Hydrol. 554, 6679 (2017). https://doi.org/10.1016/j.jhydrol.2017.08.052. http:// www.sciencedirect.com/science/article/pii/S0022169417305899

51. Nordbotten, J.M., Boon, W., Fumagalli, A., Keilegavlen, E.: Unified approach to discretization of flow in fractured porous media. Comput Geosci. https://link.springer.com/article/10.1007/ s10596-018-9778-9 (2018)
52. Pichot, G., Erhel, J., de Dreuzy, J.R.: A generalized mixed hybrid mortar method for solving flow in stochastic discrete fracture networks. SIAM J. Sci. Comput. 34(1), B86-B105 (2012). https://doi.org/10.1137/100804383

53. Pichot, G., Poirriez, B., Erhel, J., de Dreuzy, J.R.: A mortar Bdd method for solving flow in stochastic discrete fracture networks. In: Erhel, J., Gander, M.J., Halpern, L., Pichot, G., Sassi, T., Widlund, O. (eds.) Domain Decomposition Methods in Science and Engineering XXI, pp. 99-112. Springer International Publishing, Cham (2014)

54. Raviart, P.A., Thomas, J.M.: A mixed finite element method for second order elliptic problems. Lecture Notes in Mathematics 606, 292-315 (1977)

55. Roberts, J.E., Thomas, J.M.: Mixed and hybrid methods. In: Handbook of Numerical Analysis, Vol.II, Handbook of Numerical Analysis, II, pp. 523-639. North-Holland, Amsterdam (1991). 10.1016/S1570-8659(05)80041-9

56. Schön, J.H.: Physical Properties of Rocks, Handbook of Petroleum Exploration and Production, vol. 8. Elsevier, Amsterdam (2011). http://www.sciencedirect.com/science/article/pii/ S1567803211080128

57. Shewchuk, J.R.: Triangle: engineering a $2 \mathrm{~d}$ quality mesh generator and delaunay triangulator. In: Lin, M.C., Manocha, D. (eds.) Applied Computational Geometry: Towards Geometric Engineering, Lecture Notes in Computer Science, vol. 1148, pp. 203-222. Springer-Verlag (1996). From the First ACM Workshop on Applied Computational Geometry

58. Kadeethum, T., Nick, H.M., Lee, S., Ballarin, F.: Flow in porous media with low dimensional fractures by employing enriched galerkin method. Adv. Water Resour. (2020)

59. Beirão da Veiga, L., Brezzi, F., Cangiani, A., Manzini, G., Marini, L.D., Russo, A.: Basic principles of virtual element methods. Math. Models Methods Appl. Sci. 23(01), 199-214 (2013). 10.1142/S0218202512500492

60. Beirão da Veiga, L., Brezzi, F., Marini, L.D., Russo, A.: H(div) and H(curl)-conforming VEM. Numer. Math. 133(2), 303-332 (2014). https://doi.org/10.1007/s00211-015-0746-1

61. Beirão da Veiga, L., Brezzi, F., Marini, L.D., Russo, A.: The hitchhiker's guide to the virtual element method. Math. Models Methods Appl. Sci. 24(08), 1541-1573 (2014). https://doi.org/10.1142/ S021820251440003X. http://www.worldscientific.com/doi/abs/ 10.1142/S021820251440003X

62. Beirão da Veiga, L., Brezzi, F., Marini, L.D., Russo, A.: Mixed virtual element methods for general second order elliptic problems on polygonal meshes. ESAIM Math. Model Numer. Anal. 50(3), 727-747 (2016). https://doi.org/10.1051/m2an/2015067

63. Wangen, M.: Physical Principles of Sedimentary Basin Analysis. Cambridge University Press, Cambridge (2010). https://books. google.no/books?id=_pCrcEVPboYC

Publisher's note Springer Nature remains neutral with regard to jurisdictional claims in published maps and institutional affiliations. 